JOURNAL OF THE

AMERICAN MATHEMATICAL SOCIETY

Volume 14, Number 4, Pages 737-782

S 0894-0347(01)00372-1

Article electronically published on May 30, 2001

\title{
NON-AXIAL SELF-SIMILAR HOLE FILLING FOR THE POROUS MEDIUM EQUATION
}

\author{
S. B. ANGENENT AND D. G. ARONSON
}

\section{INTRODUCTION}

Self-similar solutions play an important rôle in the development of the theory of non-linear evolution equations. In addition to providing exact and sometimes even explicit solutions which can be used to validate numerical schemes, they often describe the asymptotic form of large classes of solutions in the neighborhood of some important change in behavior (such as blow-up) or at large times. Often a self-similar solution to an evolution equation in the variables $\left(x_{1}, \ldots, x_{d}, t\right)$ is a function of the form

$$
t^{\beta} F\left(\frac{x_{1}}{t^{\alpha_{1}}}, \ldots, \frac{x_{d}}{t^{\alpha_{d}}}\right)
$$

where the exponents $\alpha_{1}, \ldots, \alpha_{d}, \beta$ and the function $F$ must be determined from the equation together with appropriate boundary and initial conditions. In some problems the exponents can be obtained a priori from scaling arguments and conservation laws. Zel'dovich calls this self-similarity of the first kind. However, it is often the case that the exponents cannot be gotten a priori and must be obtained by solving what amounts to a non-linear eigenvalue problem for the function $F$. This is what Zel'dovich calls self-similarity of the second kind, and the solutions which we study in this paper are of this type. A cogent account of the theory of self-similar solutions with many illuminating examples can be found in Barenblatt's book [7].

We are concerned with the so-called focusing or hole-filling problem for the porous medium equation (PME)

$$
\partial_{t} u=\Delta\left(u^{m}\right)
$$

where $\Delta$ is the Laplace operator in $\mathbb{R}^{d}$ and $m>1$ is a constant. In the focusing problem we solve the initial value problem for equation (1.1) with data at $t=0$ whose support lies outside a compact set $K$. It is known that the support of the solution $u(\cdot, t)$ is non-decreasing with $t$, and that eventually it is strictly increasing. Thus at some finite time $T>0$ the support of $u(\cdot, T)$ will first cover all of $K$. We call $T$ the focusing time. The focusing problem is well studied in the axially symmetric case. There exists a one-parameter family of axially symmetric self-similar solutions of the second kind $([17,6])$, and some member of this family describes locally, to

Received by the editors November 1, 1999.

2000 Mathematics Subject Classification. Primary 35K65, 37G99; Secondary 35K55, 76 S05.

Key words and phrases. Porous medium equation, self-similar solutions, symmetry breaking bifurcation.

The first author was supported by the National Science Foundation. 
leading order, the behavior of essentially any focusing solution to (1.1) ([1, 2]). Here we are concerned with the existence of non-axial self-similar solutions which bifurcate from the axially symmetric solutions.

To describe and derive our results, it is convenient to change the dependent variable to

$$
v=\frac{m}{m-1} u^{m-1}
$$

and replace equation (1.1) with

$$
\partial_{t} v=(m-1) v \Delta v+|\nabla v|^{2} .
$$

In the classical setting [4], $u$ represents the scaled density of ideal gas flowing isentropically in a homogeneous porous medium and $v$ represents the scaled pressure. The self-similar solutions to (1.2) which we seek are weak solutions of the form

$$
v(x, t)=(T-t)^{2 a-1} V_{c}\left(\frac{x}{(T-t)^{a}}\right)
$$

defined for all $t \leq T$, where $c$ is a parameter and $a$ is the similarity exponent.

In view of the scaling properties of the pressure equation (1.2), we can recover the whole one-parameter family of self-similar solutions from any given one, say, $v_{1}(x, t)$. Specifically,

$$
V_{c}(\xi)=c^{2} V_{1}\left(c^{-1} \xi\right)
$$

for any $c>0$, where

$$
\xi=|x| /(T-t)^{a} .
$$

In [6] it is shown that for each $m \in(1, \infty)$ there exists a unique exponent $a_{m} \in\left(\frac{1}{2}, 1\right)$ for which a one-parameter family of radial self-similar solutions exists, i.e., solutions of the form

$$
v_{c}(x, t)=(T-t)^{2 a-1} V_{c}\left(\frac{|x|}{(T-t)^{a}}\right)
$$

with $a=a_{m}$. Moreover, there exist $\gamma_{m}, K_{m} \in(0, \infty)$ such that

$$
V_{c}(\xi)\left\{\begin{array}{l}
=0 \text { for } 0 \leq \xi \leq\left(\frac{c}{\gamma_{m}}\right)^{a} \\
>0 \text { for } \xi>\left(\frac{c}{\gamma_{m}}\right)^{a}
\end{array}\right.
$$

and

$$
V_{c}(\xi)=K_{m} c^{1 / a} \xi^{2-1 / a}+o\left(\xi^{2-1 / a}\right) \quad(\xi \rightarrow \infty) .
$$

Thus the $v_{c}$ are focusing or hole-filling solutions with the boundary of the support (i.e., the interface) given by

$$
|x|=\left(\frac{c}{\gamma_{m}}(T-t)\right)^{a} .
$$

We normalize the $v_{c}$ by setting $c=\gamma_{m}$ so that $V(\xi)=0$ on $[0,1]$ and $V(\xi)>0$ on $[1, \infty)$. We write $V$ for $V_{\gamma_{m}}$. We will refer to these solutions as the AG solutions. On the interval where $V$ is positive it is the solution to a degenerate non-linear ordinary differential equation which is obtained by substituting (1.3) in equation (1.2).

Physical experiments involving convergent gravity currents $(m=4, d=2)$ followed by numerical experiments [13] indicate that small deviations from rotational 
symmetry are amplified as the solution "tries to fill up the hole". A formal linear stability analysis [10] shows that the AG solutions are indeed unstable, at least when $m$ is close to 1 , and that the number of unstable modes increases as $m$ tends down to 1 . This suggests that a sequence of bifurcations occurs as $m$ decreases from $\infty$ to 1 .

In this paper we give a rigorous proof of the existence of these bifurcations. More specifically, we prove that as $m \searrow 1$ the axially symmetric AG self-similar solutions given by (1.3) undergo an infinite sequence of symmetry breaking bifurcations. In particular, there exist infinitely many families of non-axial focusing self-similar solutions to the porous medium equation (1.3). A more precise and technical statement of this result is given below as Theorem 1.1.

The proof of our result proceeds in several steps. First we prove that focusing self-similar solutions to the porous medium equation are solutions to a non-linear analytic Fredholm equation. We then apply the Lyapunov-Schmidt construction to obtain a finite set of analytic equations from which we deduce the existence of bifurcating solutions.

In Section 2 we examine general properties of focusing self-similar solutions. Specifically, we show that if $V$ satisfies the growth condition

$$
V(\xi)=O\left(|\xi|^{2-1 / a}\right)
$$

and if the zeroset of $V$ is bounded, then $V$ has an asymptotic expansion as $|\xi| \rightarrow \infty$. Moreover all of the level sets $V^{-1}(y)$ are smooth star-shaped compact hypersurfaces which can be represented as graphs $r=R(y, \theta)$ over the unit sphere $S^{d-1}$.

Let $v(r, \theta, t)$ be a solution to the pressure equation (1.2) written in polar coordinates, which has star-shaped level sets given in polar coordinates by $r=R(y, \theta, t)$ on $[0, \infty) \times S^{d-1} \times(0, \infty)$. We derive, in Section 3 , the partial differential equation for the evolution of $R(y, \theta, t)$. The equation for $R$ is then transformed by the introduction of similarity variables. Let

$$
\eta=\frac{y}{(T-t)^{2 a-1}} \quad \text { and } \quad \tau=-\ln (T-t)
$$

and define $\Upsilon$ by

$$
R(y, \theta, t)=(T-t)^{a} \Upsilon(\eta, \theta, \tau) .
$$

The form of the differential equation for $\Upsilon$ suggests that the variable $P=\ln \Upsilon$ is more natural, so finally we derive the evolution equation for $P(\eta, \theta, \tau)$. The advantage of using the similarity variables lies in the fact that if $v$ is a self-similar solution to (1.2), then $P$ is a stationary solution to its evolution equation. In particular, this means that in seeking self-similar solutions we must solve an elliptic equation rather than a parabolic one.

We know from the results of Section 2 that for the AG solutions

$$
e^{P} \sim \eta^{\frac{a}{2 a-1}}
$$

Since we are looking for bifurcations from the AG solutions we write

$$
P(\eta, \theta)=\frac{a}{2 a-1} L(\eta)+N(\eta, \theta),
$$

where $L(\eta)$ is a fixed positive $C^{\infty}$ function which is equal to $\ln \eta$ when $\eta$ is large, and $N$ is uniformly bounded. The elliptic equation for $N$ is degenerate at both $\eta=0$ and $\eta=\infty$. Roughly speaking, specifying $a$ fixes the growth at infinity and we seek those values of $a$ for which the equation for $N$ has a solution with 
the appropriate regularity at zero. In Section 4 we formulate our problem as a non-linear Fréchèt differentiable map $\mathfrak{f}(N, a, m)=0$ on a suitable Banach space. Sections 5, 6, and 7 are devoted to the technical proof that the Fréchèt derivative $\mathfrak{f}_{N}(N, a, m)$ of $\mathfrak{f}(N, a, m)$ with respect to $N$ is a Fredholm operator of index 0 . The main problem is to obtain the required Schauder estimates in the neighborhood of the degeneracies.

Section 8 is devoted to the study of the linearization of our problem at the AG solutions. In this setting $\mathfrak{f}_{N}$ is formally self-adjoint in a suitably weighted $L^{2}$ space so that its spectrum is real, discrete, and bounded above. The range of $\mathfrak{f}$ and of $\mathfrak{f}_{N}$ is a Banach space $X$ which is decomposed into a direct sum

$$
X=X^{0} \oplus X^{1} \oplus X^{2} \oplus \cdots
$$

where $X^{l}$ is the space of functions $\varphi(\eta, \theta)$ on $[0, \infty] \times S^{d-1}$ which can be written in the form

$$
\varphi(\eta, \theta)=\Phi_{1}(\eta) y_{l}^{(1)}(\theta)+\cdots+\Phi_{n}(\eta) y_{l}^{(n)}(\theta)
$$

with $\left\{y_{l}^{(1)}, \ldots, y_{l}^{(n)}\right\}$ an orthogonal basis for the spherical harmonics of degree $l$. The eigenvalue problem is considered on each summand $X^{l}$ and the eigenvalues $\lambda_{l j}(m)$ form a doubly infinite sequence, where the first index refers to the degree of the spherical harmonics and the second to the number of zeros of the corresponding eigenfunctions. The $\lambda_{l j}(m)$ are analytic functions of $m$. We show that, for all $m$, $\lambda_{01}=0$, and $\lambda_{l j}<0$ if $l=0$ and $j>1$ or if $l \geq 1$ and $j \geq 1$. Moreover, $\lambda_{l 0}>0$ for $l=0$ and 1 . Only the eigenvalues $\lambda_{l 0}(m)$ for $l \geq 2$ can change sign as $m$ varies. The values of $m$ for which this occurs are, therefore, the only candidates for bifurcation points. We prove that there are no bifurcations in the class of radially symmetric solutions. However, we show that there are symmetry breaking bifurcations whenever a $\lambda_{l 0}(m)$ changes sign, and solutions on the bifurcating branches do not have full rotational symmetry. The proof of these assertions is based on the LyapunovSchmidt reduction and the fact that our problem is equivariant with respect to the group $O(d, \mathbb{R})$ of $d \times d$ orthogonal matrices.

In Section 9 we consider the behavior of the AG solutions as $m \searrow 1$ and show that symmetry breaking bifurcations occur for all sufficiently large values of $l$. Specifically, we complete the proof of our main result:

Theorem 1.1. For every $\bar{m} \in(1, \infty)$ there exists an integer $l_{*}(\bar{m}) \geq 2$ such that corresponding to each integer $l>l_{*}(\bar{m})$ there is a bifurcation point $m_{l} \in(1, \bar{m})$. At $m=m_{l}$ a symmetry breaking bifurcation from the axially symmetric $A G$ solution occurs resulting in non-axial self-similar focusing solutions with $O(d-1, \mathbb{R})$ symmetry.

Functions with $O(d-1, \mathbb{R})$ symmetry can be seen as functions of two variables, say $x_{1}$ and $\rho=\sqrt{x_{2}^{2}+\cdots+x_{d}^{2}}$, i.e.,

$$
V\left(x_{1}, \ldots, x_{d}\right)=\bar{V}\left(x_{1}, \rho\right)
$$

The level sets $V=\eta$ are obtained by rotating the curves $\bar{V}\left(x_{1}, \rho\right)=\eta$ about the $x_{1}$-axis, i.e., by letting the group $O(d-1, \mathbb{R})$ act on the curves (see Figure 1). For the bifurcating solutions the curves are close to semicircles, and in polar coordinates $R=\sqrt{x_{1}^{2}+\rho^{2}}, \psi=\arctan \frac{\rho}{x_{1}}$ in the $(x, \rho)$-plane they are given by

$$
R=R_{1}(\eta)+\varepsilon R_{2}(\eta) P_{l}^{(d)}(\cos \psi)+o(\varepsilon)
$$




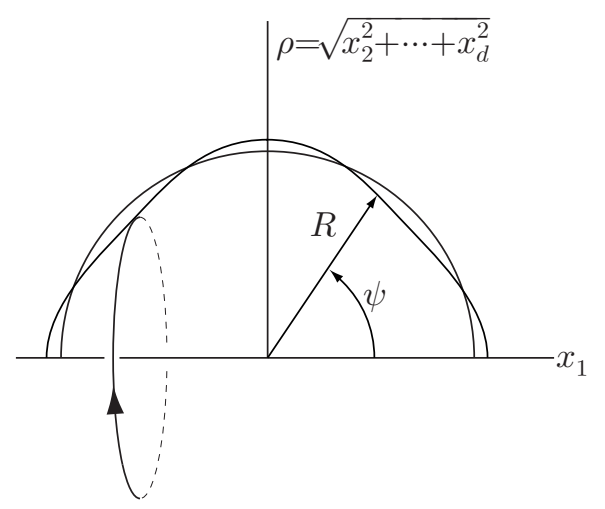

Figure 1 . The level set $\bar{V}=\eta$.

where the $R_{j}(\eta)$ depend only on $\eta$, and $\varepsilon$ is a small number which measures the distance from the bifurcation point. Here $P_{l}^{(2)}(\cos \psi)$ is the Chebyshev polynomial $\cos (l \psi), P_{l}^{(3)}(\cos \psi)$ is the usual Legendre polynomial, and, in general, $P_{l}^{(d)}(\cos \psi)$ is a Gegenbauer polynomial; the associated spherical harmonics are called zonal harmonics (cf. [14, pp.122-133] and [18]). In two dimensions $(d=2)$ the level sets $V=\eta$ are already curves and $O(1, \mathbb{R})$ symmetry means that they are symmetric with respect to reflection in the $x_{1}$-axis, and the curve $\bar{V}=\eta$ is simply the half of the curve $V=\eta$ which lies above the $x_{1}$-axis. Since $P_{l}^{(2)}(\cos \psi)=\cos (l \psi)$ the level curves $V=\eta$ also possess dihedral symmetry, i.e., they are invariant under rotation by $2 \pi / l$ radians.

Numerical studies for the case $d=2$ are described in [10] and round out the bifurcation picture. They suggest that for each $l>2$ there is a unique bifurcation point $m=m_{l}$ such that

$$
\lambda_{l 0}(m)\left\{\begin{array}{l}
>0 \text { for } m \in\left(1, m_{l}\right), \\
<0 \text { for } m \in\left(m_{l}, \infty\right),
\end{array}\right.
$$

and that the $m_{l}$ are ordered with

$$
\infty>m_{3}>m_{4}>\cdots>m_{l}>\cdots \searrow 1 .
$$

Moreover, for each $l$, the bifurcation branches appear to lie on smooth curves, extending from $m=m_{l}$ down to $m=1$. A portion of the bifurcation diagram for the similarity exponents is shown in Figure 2

Our theory gives no information about the occurrence of bifurcations for $l=2$, and the numerical evidence strongly indicates that there are no such bifurcations, i.e., that the AG solutions are linearly unstable with respect to perturbations with wave number 2 for all values of $m$. Further numerical investigations of this case are reported in [11].

As we observed above, the AG solutions describe the leading term of the asymptotic form of any axially symmetric focusing solution to equation (1.2). It is natural to ask if the $l$-fold symmetric self-similar solutions whose existence is established in this paper play a similar rôle. There is, as yet, no theoretical answer to this question, but very detailed numerical studies carried out in [10] strongly suggest an affirmative answer. The results of [10] show that, at least in the plane case $d=2$, 


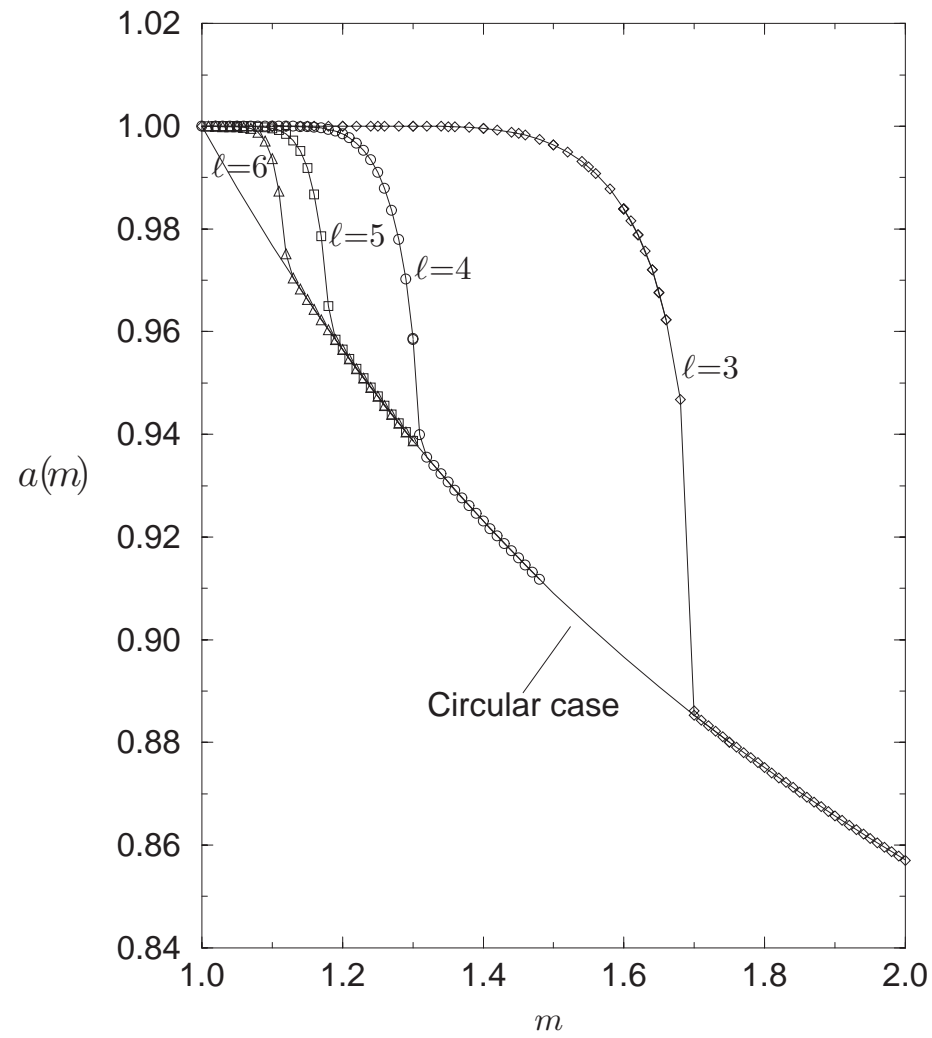

Figure 2. Computed bifurcation diagram for $d=2$ showing for $l=3, \ldots, 6$ the branches of the similarity exponents $a_{l}(m)$ corresponding to self-similar solutions with $l$-fold dihedral symmetry which bifurcate from the rotationally symmetric AG-branch. (Adapted from [10].)

there is a unique bifurcation value of $m$ for each $l>2$, and that the bifurcating solutions occur on smooth curves in the $(m, a)$-plane.

\section{Self-Similarity in General}

Formally a function $V(\xi)$ generates a self-similar solution of (1.2) if it satisfies

$$
a \xi \cdot \nabla V-(2 a-1) V=(m-1) V \Delta V+|\nabla V|^{2} .
$$

Rigorously we say a function $V(\xi)$ is a self-similar solution of PME if the associated function $v(x, t)$ defined by (1.3) is a weak solution to PME. In particular, self-similar solutions are Hölder continuous functions and they are smooth solutions of (2.1) in the region where they are positive.

From (1.2) one sees that once one has a solution $V(\xi)$, then the functions

$$
V^{\lambda}(\xi)=\lambda^{-2} V(\lambda \xi) \quad(\forall \lambda>0)
$$

also define self-similar solutions. 
Lemma 2.1. Let $V$ be a continuous self-similar solution which satisfies

$$
A_{1}|\xi|^{2-1 / a} \leq V(\xi) \leq A_{2}|\xi|^{2-1 / a}
$$

for all $\xi$ with $|\xi|$ sufficiently large, and for certain constants $0<A_{1}<A_{2}$ which do not depend on $\xi$. Then $V(\xi)$ has an asymptotic expansion for $|\xi| \rightarrow \infty$ of the form

$$
V(r \theta)=r^{2-1 / a}\left\{V^{(0)}(\theta)+r^{-1 / a} V^{(1)}(\theta)+r^{-2 / a} V^{(2)}(\theta)+r^{-3 / a} V^{(3)}(\theta)+\cdots\right\}
$$

in which the $V^{(j)}(\theta)$ are $C^{\infty}$ smooth functions on $S^{d-1}$ with $V^{(0)}(\theta)>0$.

This expansion can be differentiated arbitrarily often.

Proof. The function $v(x, t)$ defined by (1.3) is strictly positive on the annulus $\frac{1}{2} \leq$ $|x| \leq 2$ for all $t \in(-\delta, 0)$ if one chooses $\delta>0$ small enough. In fact on this annulus it is both bounded and bounded away from zero, so that there it is a solution to a non-degenerate parabolic PDE, and hence satisfies interior estimates. All its derivatives are thus uniformly bounded for $\frac{2}{3} \leq|x| \leq \frac{3}{2}$ and $\tau \in(-\delta / 2,0)$. The

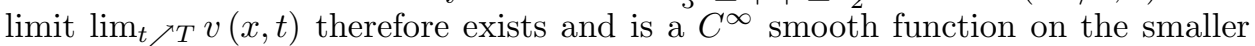
annulus $\frac{3}{4} \leq|x| \leq \frac{4}{3}$. By Taylor's theorem we have

$$
v(x, t)=\sum_{j=0}^{N}(-1)^{j} \partial_{t}^{j} v(x, T) \frac{(T-t)^{j}}{j !}+\mathcal{O}\left((T-t)^{N+1}\right) \quad(t \nearrow T)
$$

and this expansion can be differentiated arbitrarily often with respect to $x$ and $t$.

Now set $r=(T-t)^{-a}, x=\theta$, and use (1.3) to get

$$
\begin{aligned}
V(r \theta) & =r^{(2 a-1) / a} v\left(\theta, T-r^{-1 / a}\right) \\
& =r^{2-1 / a} \sum_{j=0}^{N}(-1)^{j} \partial_{t}^{j} v(\theta, T) \frac{r^{-j / a}}{j !}+\mathcal{O}\left(r^{-(N+1) / a}\right) \quad(r \nearrow \infty)
\end{aligned}
$$

as claimed.

2.1. Growth at infinity for subquadratic self-similar solutions. Let $V(\xi)$ be a self-similar solution such that for any $\varepsilon>0$ a $C_{\varepsilon}<\infty$ exists for which

$$
V(\xi) \leq C_{\varepsilon}+\varepsilon|\xi|^{2} .
$$

Then the solution $v(x, t)=(1-t)^{2 a-1} V\left(x(1-t)^{-a}\right)$ of PME has its initial data bounded by $C_{\varepsilon}+\varepsilon|x|^{2}$ and thus remains uniformly bounded for $|x| \leq 1$ and $0 \leq$ $t \leq 1$ (by comparison with explicit solutions of the form $\bar{v}(x, t)=C(t)+\varepsilon(t)|x|^{2}$ ). Setting $x=\theta \in S^{d-1}$ and $r=(1-t)^{-a}$ we conclude from $v(x, t) \leq A$ for $|x| \leq 1$ and $0 \leq t \leq 1$ that

$$
V(r \theta) \leq A r^{2-1 / a}
$$

2.2. Consequences of the Aronson-Benilan inequality. Let $V(\xi)$ be a selfsimilar solution whose corresponding solution $v(x, t)$ can be extended to $t=T$, or, which satisfies

$$
V(\xi) \leq A_{2}|\xi|^{2-1 / a}
$$


for some suitably large constant $A_{2}$. Thus $v$ is a solution of the PME for all $t \in(-\infty, T)$ and the Aronson-Benilan inequality ([3] $)$ implies that

$$
v_{t} \geq-\frac{C}{t-t_{0}} \quad\left(t_{0}<t<T\right)
$$

for any $t_{0} \in(-\infty, T)$. We may in particular let $t_{0} \rightarrow-\infty$ which shows that any self-similar solution must be a subsolution. In light of (1.3) this is equivalent to

$$
a \xi \cdot \nabla V(\xi)-(2 a-1) V(\xi) \geq 0 .
$$

The latter inequality can be written as

$$
\frac{\partial}{\partial r}\left(\frac{V(r \theta)}{r^{2-1 / a}}\right) \geq 0
$$

so we see that for any self-similar solution $V(\xi)$ of exponent $a$ which satisfies the growth condition (2.5) the zeroset $V^{-1}(0)$ is star-shaped around the origin, and outside the zeroset one has

$$
V(r \theta) \geq\left(\frac{r}{r_{0}}\right)^{2-1 / a} V\left(r_{0} \theta\right) \quad\left(\forall r \geq r_{0}, \theta \in S^{d-1}\right) .
$$

If the zeroset is bounded, then, by Hölder continuity of self-similar solutions, one has

$$
\inf _{\theta \in S^{d-1}} V(R \theta)>0
$$

for large enough $R>0$ and hence $V(\xi)$ must also satisfy a lower bound of the type $V(\xi) \geq A_{1}|\xi|^{2-1 / a}$ for some $A_{1}>0$ and for all $\xi$ with $|\xi| \geq R$. We have proved:

Lemma 2.2. Any self-similar solution which satisfies a growth condition (2.5) and whose zeroset is bounded also satisfies a lower bound $V(\xi) \geq A_{1}|\xi|^{2-1 / a}$ and by Lemma 2.1 also has an asymptotic expansion of the form (2.3). has

A further consequence of (2.6) is that at any point $\xi=r \theta$ where $V(\xi)>0$ one

$$
\frac{\partial V(r \theta)}{\partial r} \geq \frac{2-1 / a}{r} V(r \theta)>0
$$

By the implicit function theorem all level sets of the self-similar solution $V$ are therefore smooth (even analytic) star-shaped hypersurfaces. If we assume that $V$ satisfies the growth condition (2.5) and that the zeroset of $V$ is bounded, then all level sets $V^{-1}(y)$ are also compact (since $V(\xi) \rightarrow \infty$ as $|\xi| \rightarrow \infty$ ). They can therefore be represented in polar coordinates as graphs $r=R(y, \theta)$ over the unit sphere $S^{d-1}$. Below we work out the equations which such a function $R$ must satisfy if its graph is to generate a self-similar solution.

\section{LEVEL SETS IN POLAR COORDinATES}

We consider a general (not necessarily self-similar) solution of the pressure equation whose level sets are star-shaped and represent the level set $\Gamma_{y}(t)=\{x \mid$ $v(x, t)=y\}$ in polar or spherical coordinates. Thus let $\Gamma_{y}(t)$ be given by

$$
r=R(y, \theta, t), \quad \theta \in S^{d-1},
$$

so that we have

$$
v(R(y, \theta, t), \theta, t)=y
$$


for all $y \geq 0, \theta \in S^{d-1}$, and $t>0$ (here we have written $v$ as a function of the polar coordinates $r$ and $\theta$ ). The porous medium equation in polar coordinates is

$$
v_{t}=(m-1) v\left\{v_{r r}+\frac{d-1}{r} v_{r}\right\}+\left(v_{r}\right)^{2}+\frac{v_{\theta}^{2}}{r^{2}} .
$$

To derive an equation for $R(y, \theta, t)$ we first relate the derivatives of $v$ and $R$ by differentiating (3.1). In doing these computations we choose coordinates $\left\{\theta_{i}\right\}_{1 \leq i \leq d-1}$ on a part of the sphere $S^{d-1}$.

We get for the first derivatives

$$
v_{r} R_{y}=1, \quad v_{\theta_{i}}+v_{r} R_{\theta_{i}}=0 .
$$

Differentiating again we find for the second derivatives

$$
\begin{aligned}
v_{r r}\left(R_{y}\right)^{2}+v_{r} R_{y y} & =0, \\
v_{r \theta_{i}} R_{y}+v_{r r} R_{y} R_{\theta_{i}}+v_{r} R_{y \theta_{i}} & =0, \\
v_{\theta_{i} \theta_{j}}+v_{\theta_{i} r} R_{\theta_{j}}+v_{\theta_{j} r} R_{\theta_{i}}+v_{r r} R_{\theta_{i}} R_{\theta_{j}}+v_{r} R_{\theta_{i} \theta_{j}} & =0 .
\end{aligned}
$$

Hence we get

$$
|\nabla v|^{2}=\left(1+\frac{\left|R_{\theta}\right|^{2}}{R^{2}}\right) \frac{1}{R_{y}^{2}}
$$

and

$$
\begin{aligned}
\Delta v & =v_{r r}+\frac{d-1}{r} v_{r}+\frac{1}{r^{2}} g^{i j} v_{\theta_{i} \theta_{j}} \\
& =-\left(1+\frac{\left|R_{\theta}\right|^{2}}{R^{2}}\right) \frac{R_{y y}}{R_{y}^{3}}+\frac{2 R_{\theta_{i}} R_{y \theta_{i}}}{R^{2} R_{y}^{2}}-\frac{\Delta_{\theta} R}{R^{2} R_{y}}+\frac{d-1}{R R_{y}} .
\end{aligned}
$$

Using $R_{t}=-v_{t} R_{y}$ we therefore find that (1.2) and (3.2) are equivalent with

$$
\begin{aligned}
\frac{\partial R}{\partial t}=(m-1) y\left\{\left(1+\frac{\left|R_{\theta}\right|^{2}}{R^{2}}\right) \frac{R_{y y}}{R_{y}^{2}}-\frac{2 R_{\theta_{i}} R_{y \theta_{i}}}{R^{2} R_{y}}+\frac{\Delta_{\theta} R}{R^{2}}-\frac{d-1}{R}\right\} & -\left\{1+\frac{\left|R_{\theta}\right|^{2}}{R^{2}}\right\} \frac{1}{R_{y}} .
\end{aligned}
$$

Here $g_{i j}$ is the metric on the sphere in the $\theta_{i}$ coordinates, $g^{i j}$ are the coefficients of the inverse matrix of $g_{i j}$, and $\Delta_{\theta}$ is the spherical Laplacian

$$
\Delta_{\theta} \varphi=g^{i j} \frac{\partial^{2} \varphi}{\partial \theta_{i} \partial \theta_{j}}+\frac{1}{2} g^{i j} \frac{\partial \log g}{\partial \theta_{i}} \frac{\partial \varphi}{\partial \theta_{j}}, \quad g=\operatorname{det}\left(g_{i j}\right) .
$$

In two dimensions we can take $\theta$ to be the angular variable in ordinary polar coordinates, in which case (3.6) reduces to

$$
\Delta_{\theta}=\left(\frac{\partial}{\partial \theta}\right)^{2} .
$$

Equation (3.5) can be written in divergence form as

$$
\begin{aligned}
\frac{\partial R}{\partial t}=(m-1) y\left[\partial_{y}\left(\left(1+R_{\theta}^{2} / R^{2}\right) \frac{-1}{R_{y}}\right)+\right. & \left.\Delta_{\theta}\left(\frac{-1}{R}\right)\right] \\
& -\frac{(m-1)(d-1) y}{R}-\frac{1+R_{\theta}^{2} / R^{2}}{R_{y}} .
\end{aligned}
$$


3.1. Similarity variables. Introduce new coordinates $\eta$ and $\tau$, given by

$$
\eta=\frac{y}{(T-t)^{2 a-1}}, \quad \tau=-\ln (T-t),
$$

and define $\Upsilon$ by

$$
R(y, \theta, t)=(T-t)^{a} \Upsilon\left(\frac{y}{(T-t)^{2 a-1}}, \theta,-\ln (T-t)\right) .
$$

The function $\Upsilon$ describes our hole-filling solution as seen in similarity coordinates. A self-similar solution $R$ will generate a time $(\tau)$ independent profile $\Upsilon$. The graph

$$
\operatorname{graph}(\Upsilon)=\left\{(\Upsilon(\eta, \theta) \theta, \eta): \eta \geq 0, \theta \in S^{d-1}\right\} \subset \mathbb{R}^{d+1}
$$

coincides with the graph

$$
\operatorname{graph}(V)=\left\{(\xi, V(\xi)): \xi \in \mathbb{R}^{d}\right\} \subset \mathbb{R}^{d+1}
$$

of a self-similar solution as discussed in Section 2, $\Upsilon$ and $V$ are related via

$$
V(\Upsilon(\eta, \theta) \theta)=\eta \text {. }
$$

Direct computation gives the following equation for $\Upsilon$ :

$$
\begin{aligned}
\frac{\partial \Upsilon}{\partial t}=(m-1) y\left[\partial_{y}(\right. & \left.\left.\left(1+\Upsilon_{\theta}^{2} / \Upsilon^{2}\right) \frac{-1}{\Upsilon_{y}}\right)+\Delta_{\theta}\left(\frac{-1}{\Upsilon}\right)\right] \\
& -\frac{(m-1)(d-1) y}{\Upsilon}-\frac{1+\Upsilon_{\theta}^{2} / \Upsilon^{2}}{\Upsilon_{y}}-(2 a-1) \eta \Upsilon_{\eta}+a \Upsilon .
\end{aligned}
$$

In view of the form of this equation it is more convenient to work with the quantity

$$
P=\ln \Upsilon
$$

which satisfies

$$
\begin{aligned}
e^{2 P} \frac{\partial P}{\partial \tau}=(m-1) \eta\left[\partial_{\eta}\right. & \left.\left(-\frac{1+P_{\theta}^{2}}{P_{\eta}}\right)+\Delta_{\theta} P\right] \\
& -d(m-1) \eta-\frac{1+P_{\theta}^{2}}{P_{\eta}}-e^{2 P}\left[(2 a-1) \eta P_{\eta}-a\right] .
\end{aligned}
$$

The equation for self-similar solutions is

$$
\begin{aligned}
0=\eta\left[\left(1+P_{\theta}^{2}\right) \frac{P_{\eta \eta}}{P_{\eta}^{2}}-\right. & \left.2 \frac{P_{\theta} P_{\theta \eta}}{P_{\eta}}+\Delta_{\theta} P\right] \\
& -d \eta-\frac{1}{m-1}\left\{\frac{1+P_{\theta}^{2}}{P_{\eta}}+e^{2 P}\left[(2 a-1) \eta P_{\eta}-a\right]\right\} .
\end{aligned}
$$

3.2. Asymptotics of $\Upsilon$ and $P$ for $\eta \rightarrow \infty$. The asymptotic expansion in Lemma 2.1 immediately translates into a similar expansion for $\Upsilon$ and $P$. The growth hypothesis (2.5) is equivalent to a lower bound on the growth of, say, $\Upsilon$ :

$$
\Upsilon(\eta, \theta) \geq A_{2}^{\prime} \eta^{a /(2 a-1)} .
$$

If this growth condition is satisfied, then $\Upsilon$ must have an expansion of the form

$$
\Upsilon(\eta, \theta) \sim \eta^{a /(2 a-1)} \sum_{j=0}^{\infty} \Upsilon^{(j)}(\theta) \eta^{-j /(2 a-1)} \quad(\eta \rightarrow \infty)
$$


Taking logarithms one gets

$$
P(\eta, \theta) \sim \frac{a}{2 a-1} \ln \eta+\sum_{j=0}^{\infty} P^{(j)}(\theta) \eta^{-j /(2 a-1)} \quad(\eta \rightarrow \infty) .
$$

\section{Formulation as a nON-Linear Fredholm equation}

Since the expected asymptotics for $\eta \rightarrow \infty$ of any solution is $e^{P} \sim \eta^{\frac{a}{2 a-1}}$ we write $P$ in the form

$$
P(\eta, \theta)=c L(\eta)+N(\eta, \theta), \quad \text { with } c=\frac{a}{2 a-1},
$$

where $L(\eta)$ is a $C^{\infty}$ function satisfying

$$
\begin{aligned}
L(\eta) & =\ln \eta \text { for } \eta \geq e^{2}, \\
L(\eta) & =\frac{1-(1-\eta)^{2 a}}{2 a} \text { for sufficiently small } \eta, \\
L^{\prime \prime}(\eta) & >0 \text { for all } \eta,
\end{aligned}
$$

and $N$ is a uniformly bounded function.

Substitute (4.1) in (3.9) and multiply the resulting equation with $L^{\prime}(\eta)$ to get

$$
\begin{aligned}
0=\eta L^{\prime}(\eta)[(1+ & \left.\left.N_{\theta}^{2}\right) \frac{N_{\eta \eta}}{\left(c L^{\prime}(\eta)+N_{\eta}\right)^{2}}-2 \frac{N_{\theta} N_{\theta \eta}}{c L^{\prime}(\eta)+N_{\eta}}+\Delta_{\theta} N\right] \\
& +\eta L^{\prime}(\eta)\left[L^{\prime \prime}(\eta) \frac{1+N_{\theta}^{2}}{\left(c L^{\prime}(\eta)+N_{\eta}\right)^{2}}-d\right] \\
& -(m-1)^{-1} \frac{L^{\prime}(\eta)\left(1+N_{\theta}^{2}\right)}{c L^{\prime}(\eta)+N_{\eta}} \\
& -(m-1)^{-1} e^{2 c L+2 N} a L^{\prime}(\eta)\left(\eta L_{\eta}-1\right) \\
& -\frac{2 a-1}{m-1} e^{2 c L+2 N} \eta L^{\prime}(\eta) N_{\eta} .
\end{aligned}
$$

The equation is degenerate elliptic both at $\eta=0$ and at $\eta=\infty$. To handle the degeneracy at $\eta=\infty$ it turns out to be useful to introduce a new independent variable $z \in[-1,0]$, given by

$$
z=-L^{\prime}(\eta)^{2 c-1}
$$

In the new $z$ variable the equation (4.3) becomes

$$
\begin{aligned}
\bar{a}_{00}\left(z, N_{\theta}, N_{z}\right) \frac{\partial^{2} N}{\partial z^{2}} & +2 \bar{a}_{0 i}\left(z, N_{\theta}, N_{z}\right) \frac{\partial^{2} N}{\partial z \partial \theta_{i}} \\
& +\bar{a}_{i j}(z, \theta) \frac{\partial^{2} N}{\partial \theta_{i} \partial \theta_{j}}+f\left(z, N, N_{z}, N_{\theta_{i}}\right)=0
\end{aligned}
$$

for suitable functions $\bar{a}_{\ldots}\left(z, N_{\theta}, N_{z}\right)$ whose precise form we shall not determine in general. It is however clear that this equation is a quasilinear elliptic equation (ellipticity being invariant under coordinate changes) which degenerates at $z=$ $-1(\eta=0)$ and at $z=0(\eta=\infty)$. The function $f\left(z, N, N_{z}, N_{\theta_{i}}\right)$ contains all terms on the last four lines in (4.3), i.e. the "lower order terms". 
4.1. Form of the equation at $z=-1(\eta=0)$. Our coordinate transformation is such that for small values of $\eta>0$ one has $\eta=1+z$. Thus (4.3) for $z$ close to -1 reads

$$
\begin{aligned}
0=(1+z)( & -z)^{2 a-1}\left[\frac{1+N_{\theta}^{2}}{\left(c(-z)^{2 a-1}+N_{z}\right)^{2}} N_{z z}-\frac{2 N_{\theta}}{c(-z)^{2 a-1}+N_{z}} N_{\theta z}+\Delta_{\theta} N\right] \\
& -(1+z)(-z)^{2 a-1}\left[(2 a-1)(-z)^{2 a-2} \frac{1+N_{\theta}^{2}}{\left(c(-z)^{2 a-1}+N_{z}\right)^{2}}+d\right] \\
& -(m-1)^{-1} \frac{(-z)^{2 a-1}\left(1+N_{\theta}^{2}\right)}{c(-z)^{2 a-1}+N_{z}} \\
& -\frac{a}{m-1} e^{2 c L+2 N}(-z)^{2 a-1}\left((1+z)(-z)^{2 a-1}-1\right) \\
& -\frac{2 a-1}{m-1} e^{2 c L+2 N}(1+z)(-z)^{2 a-1} N_{z} .
\end{aligned}
$$

4.2. Form of the equation at $z=0(\eta=\infty)$. For $\eta \geq e^{2}$, i.e. for $-e^{-2(2 c-1)} \leq$ $z<0$, one has

$$
z=-\eta^{-(2 c-1)}, \quad \text { and also } L^{\prime}(\eta)=\frac{1}{\eta}
$$

so

$$
z \partial_{z}=-(2 a-1) \eta \partial_{\eta} \quad \text { and } \quad \eta L^{\prime}(\eta)-1=0 .
$$

Thus (4.3) in the region $-1 \ll z<0$ is

$$
\begin{aligned}
0=\frac{1+N_{\theta}^{2}}{\left(a-z N_{z}\right)^{2}} z^{2} & \frac{\partial^{2} N}{\partial z^{2}}-\frac{2 N_{\theta}}{a-z N_{z}} z \frac{\partial^{2} N}{\partial z \partial \theta}+\Delta_{\theta} N \\
& +\frac{2 a\left(1+N_{\theta}^{2}\right)}{\left(a-z N_{z}\right)^{2}} z \frac{\partial N}{\partial z} \\
& +\frac{(2 a-1)^{2}\left(1+N_{\theta}^{2}\right)}{\left(a-z N_{z}\right)^{2}}-d \\
& -\frac{1}{m-1} \frac{(2 a-1)^{2}\left(1+N_{\theta}^{2}\right)}{a-z N_{z}} \\
& -\frac{2 a-1}{m-1} e^{2 N} \frac{\partial N}{\partial z} .
\end{aligned}
$$


4.3. General form reflecting the degeneracy. We can rewrite the general equation in the form

$$
\begin{aligned}
& (1+z)\left\{z^{2} a_{00}\left(z, N_{\theta}, z N_{z}\right) \frac{\partial^{2} N}{\partial z^{2}}+2 z a_{0 i}\left(z, N_{\theta}, z N_{z}\right) \frac{\partial^{2} N}{\partial z \partial \theta_{i}}\right. \\
& \left.+a_{i j}(z, \theta) \frac{\partial^{2} N}{\partial \theta_{i} \partial \theta_{j}}\right\}+f\left(z, N, N_{z}, N_{\theta_{i}}\right)=0
\end{aligned}
$$

where the coefficients $a_{\ldots}$.. are now smooth functions of their arguments satisfying a uniform ellipticity condition

$$
\delta \leq \frac{a_{00} \zeta^{2}+2 a_{0 i} \zeta \lambda_{i}+a_{i j} \lambda_{i} \lambda_{j}}{\zeta^{2}+|\lambda|^{2}} \leq \delta^{-1} \quad \text { for all }\left(\zeta, \lambda_{i}\right) \in \mathbb{R}^{d}
$$

for some $\delta=\delta\left(z, \theta, N_{\theta}, z N_{z}\right)>0$.

4.4. Asymptotics of $P$ and $N$ in the $z$ variable. For large $\eta$ one has $z=$ $-\eta^{-1 /(2 a-1)}$ so that we can rewrite the asymptotic expansions (3.11) and (3.10) as

$$
\begin{array}{ll}
\Upsilon(z, \theta) \sim(-z)^{a} \sum_{j=0}^{\infty} \Upsilon^{(j)}(\theta)(-1)^{j} z^{j} & (z \nearrow 0) \\
P(z, \theta) \sim a \ln (-z)+\sum_{j=0}^{\infty} P^{(j)}(\theta)(-1)^{j} z^{j} & (z \nearrow 0),
\end{array}
$$

and

$$
N(z, \theta) \sim \sum_{j=0}^{\infty} P^{(j)}(\theta)(-1)^{j} z^{j} \quad(z \nearrow 0) .
$$

Again these expansions can be differentiated any number of times.

4.5. The non-linear differential operator and spaces between which it acts. We define $Q=[-1,0] \times S^{d-1}$ and introduce the Banach space

$$
X=h^{0, \alpha}(Q)
$$

as the closure of $C^{\infty}(Q)$ in the norm

$$
\|u\|_{X}=\sup _{Q}|u(z, \theta)|+\sup _{\theta \neq \theta^{\prime}} \frac{\left|u(z, \theta)-u\left(z, \theta^{\prime}\right)\right|}{\left|\theta-\theta^{\prime}\right|^{\alpha}} .
$$

Thus functions $u \in X$ are uniformly Hölder continuous in the angle variable $\theta$, but not necessarily in the radial variable $z$.

Within the space $X$ we distinguish the smaller subspace $Y$ of functions $u \in X$ for which

$$
(1+z) z^{2} u_{z z},(1+z) z u_{z \theta_{i}},(1+z) u_{\theta_{i} \theta_{j}}, u_{z}, u_{\theta_{i}} \in X .
$$

With the norm

$$
\begin{aligned}
\|u\|_{Y}= & \left\|(1+z) z^{2} u_{z z}\right\|_{X}+\left\|(1+z) z u_{z \theta_{i}}\right\|_{X}+\left\|(1+z) u_{\theta_{i} \theta_{j}}\right\|_{X} \\
& +\left\|u_{z}\right\|_{X}+\left\|u_{\theta_{i}}\right\|_{X},
\end{aligned}
$$

$Y$ is also a Banach space. 
The equation for self-similar focusing solutions for the porous medium equation can be written as an equation on $Y \times \mathbb{R}^{2}$ of the form

$$
\mathfrak{f}(N, a, m)=0,
$$

where, by definition, $\mathfrak{f}(N, a, m)$ is the left-hand side of [4.6). The domain of this map is

$$
\mathcal{O}=\left\{(N, a, m): c L^{\prime}(\eta)+N_{\eta} \geq \delta \text { for some } \delta>0\right\} .
$$

(Here $z=-L^{\prime}(\eta)^{2 c-1}$ and $c=a /(2 a-1)$ are understood.)

Lemma 4.1. Let $V(\xi)$ be a self-similar solution of PME which satisfies the conditions of Section 2 and for which $|\nabla V(\xi)| \geq \delta>0$ for all $\xi$ near the free boundary with $V(\xi)>0$. Let $P(\eta, \theta)$ and $N(\eta, \theta)$ be defined as above. Then $N \in Y$.

Proof. The lower bound on the gradient of the solution near the free boundary implies that the free boundary is smooth, and that $V(\xi)$ is a smooth function near the free boundary. Consequently $\Upsilon, P$, and $N$ are smooth functions near $\eta=0$. For $0<\eta<\infty$ the function $N$ is also smooth, and the asymptotic expansion (4.10) shows that $N$ is sufficiently smooth near $z=0$ (i.e. $\eta=\infty$ ) for $N$ to belong to the class $Y$.

This lemma tells us that all reasonable self-similar solutions correspond to solutions of $\mathfrak{f}(N, a, m)=0$ and that $\mathcal{O}$ is the right place to look for solutions of (4.6).

Theorem 4.2. The map $\mathfrak{f}: \mathcal{O} \rightarrow X$ is $C^{\omega}$ Fréchèt differentiable. The Fréchèt derivative of $\mathfrak{f}(N, a, m)$ with respect to $N$ is a Fredholm operator of index 0.

Fréchèt differentiability of the map $\mathfrak{f}$ is the easy part, as it follows from the (Fréchèt) smoothness of substitution operators $u \in h^{\alpha} \longmapsto \varphi \circ u \in h^{\alpha}$ whenever $\varphi$ is smooth. In fact, if $\varphi$ is analytic, then the substitution operator is also analytic. We will not compute the Fréchèt derivatives with respect to $N, a$, and $m$ explicitly, but only observe that the derivative in $N$ is given by the second order elliptic operator

$$
\begin{aligned}
\frac{\partial \mathfrak{f}}{\partial N} \cdot \nu=(1+z) z^{2} A_{00}(z, \theta) \frac{\partial^{2} \nu}{\partial z^{2}} & +2(1+z) z A_{0 i}(z, \theta) \frac{\partial^{2} \nu}{\partial z \partial \theta_{i}} \\
+(1+z) A_{i j}(z, \theta) \frac{\partial^{2} \nu}{\partial \theta_{i} \partial \theta_{j}} & \\
& +k_{0}(z, \theta) \frac{\partial \nu}{\partial z}+k_{i}(z, \theta) \frac{\partial \nu}{\partial \theta_{i}}+\ell(z, \theta) \nu
\end{aligned}
$$

where the second order terms have coefficients

$$
\begin{gathered}
A_{00}(z, \theta)=a_{00}\left(z, N_{\theta}, z N_{z}\right), \\
A_{0 i}(z, \theta)=a_{0 i}\left(z, N_{\theta}, z N_{z}\right), \\
A_{i j}(z, \theta)=a_{i j}(z, \theta)
\end{gathered}
$$


(note that the $a_{i j}$ are the coefficients of the spherical Laplacian and hence do not depend on $z, N$, or its derivatives). The lower order terms are

$$
\begin{aligned}
k_{0}(z, \theta)=\frac{\partial f}{\partial N_{z}}\left(z, N, N_{z}, N_{\theta_{i}}\right)+(1+z) z^{2} N_{z z} \frac{\partial a_{00}\left(z, N_{\theta}, z N_{z}\right)}{\partial N_{z}} & \\
& +2(1+z) z N_{z \theta_{i}} \frac{\partial a_{0 i}\left(z, N_{\theta}, z N_{z}\right)}{\partial N_{z}}, \\
k_{i}(z, \theta)=\frac{\partial f}{\partial N_{\theta_{i}}}\left(z, N, N_{z}, N_{\theta_{i}}\right)+(1+z) z^{2} N_{z z} \frac{\partial a_{00}\left(z, N_{\theta}, z N_{z}\right)}{\partial N_{\theta_{i}}} & \\
& +2(1+z) z N_{z \theta_{i}} \frac{\partial a_{0 i}\left(z, N_{\theta}, z N_{z}\right)}{\partial N_{\theta_{i}}},
\end{aligned}
$$

and finally

$$
\ell(z, \theta)=\frac{\partial f}{\partial N}\left(z, N, N_{z}, N_{\theta_{i}}\right)
$$

To establish the Fredholm property for this operator from $Y \rightarrow X$ we will prove:

Theorem 4.3. Let $M$ be a differential operator given by the right-hand side of (4.11), and assume

(1) its coefficients belong to $h^{0, \alpha}\left([-1,0] \times S^{d-1}\right)$,

(2) the coefficients $A_{00}, A_{0 i}$, and $A_{i j}$ are uniformly elliptic (i.e. they satisfy (4.7),

(3) the "z-drift coefficient" $k_{0}(z, \theta)$ satisfies

$$
k_{0}(-1, \theta)>0>k_{0}(0, \theta)
$$

for all $\theta \in S^{d-1}$.

Then $M: Y \rightarrow X$ is a Fredholm operator of index 0 .

If $\ell(z, \theta)<0$, then $M$ is invertible.

The proof, which we give in the sections below, will follow the beaten path of "a priori estimates and continuity" where, as usual, the a priori estimates will require most of our efforts. Here we will merely verify that the operator $\mathfrak{f}_{N}$ satisfies the conditions of the theorem.

The only non-obvious condition is the third, about the drift term, and it even requires a lemma:

Lemma 4.4. If $u \in Y$, then

$$
\lim _{z \nearrow 0} z^{2} u_{z z}=\lim _{z \nearrow 0} z u_{z \theta_{i}}=\lim _{z \searrow-1}(1+z) u_{z}=\lim _{z \searrow-1}(1+z) u_{z z}=\lim _{z \searrow-1}(1+z) u_{z \theta}=0 .
$$

Proof. By definition the functions $z(1+z) u_{z \theta_{i}}$, etc. all belong to $h^{0, \alpha}$ so their values at $z=0$ or $z=-1$ are well defined, and the limits must exist. If, to pick one, $z(1+z) u_{z \theta_{i}}$ would not vanish at $z=0$, then for some non-zero function $U(\theta) \in h^{\alpha}\left(\mathbb{R}^{d-1}\right)$ one would have $u_{z \theta_{i}}=z^{-1} U(\theta)+o\left(z^{-1}\right)$ which would imply $u_{\theta_{i}}(z, \theta)=U(\theta) \ln |z|+o(\ln |z|)$. But this would contradict the fact that $u_{\theta_{i}}$ is bounded for any $u \in Y$. The same arguments apply to the other limits. 
Since $N \in Y$ the quantities $(1+z) z^{2} N_{z z}$ and $(1+z) z N_{z \theta_{i}}$ vanish at $z=-1$ and $z=0$ so we have

$$
k_{0}(z, \theta)=\frac{\partial f}{\partial N_{z}}\left(z, N, N_{z}, N_{\theta_{i}}\right) \quad \text { if } z=0 \text { or } z=-1 .
$$

At $z=-1$ we get

$$
f\left(-1, N, N_{z}, N_{\theta}\right)=-(m-1)^{-1} \frac{1+N_{\theta}^{2}}{c+N_{z}}+\frac{a}{m-1} e^{2 N},
$$

which implies

$$
k_{0}(-1, \theta)=(m-1)^{-1} \frac{1+N_{\theta}^{2}}{\left(c+N_{z}\right)^{2}}>0,
$$

while at $z=0$ we get

$$
f\left(0, N, N_{z}, N_{\theta}\right)=\frac{(2 a-1)^{2}\left(1+N_{\theta}^{2}\right)}{a^{2}}-d-\frac{(2 a-1)^{2}\left(1+N_{\theta}^{2}\right)}{a}-\frac{2 a-1}{m-1} e^{2 N} \frac{\partial N}{\partial z}
$$

implying that

$$
k_{0}(0, \theta)=-\frac{2 a-1}{m-1} e^{2 N}<0 .
$$

\section{Schauder estimates AT $z=0$}

Let $L$ be the differential operator

$$
L=a z^{2} \partial_{z}^{2}+2 b z \partial_{z} \partial_{\theta}+c \Delta_{\theta}-k \partial_{z},
$$

where $a, b, c$, and $k$ are constants which satisfy

$$
a c-b^{2}>0 \quad \text { and } \quad k>0 .
$$

We assume here that the angle $\theta$ takes values $\mathbb{R}^{d-1}$ rather than the $d-1$ dimensional sphere. This does not matter since $L$ is only a local model of the general variable coefficient operator we wish to study.

The inhomogeneous equation $L u=f$ is of the form

$$
a z^{2} N_{z z}+2 b z N_{z \theta}+c \Delta_{\theta} N-k N_{z}=f,
$$

where the coefficients $a, b, c, k$ and the forcing term $f$ are such that the equation is elliptic for $-1<z<0\left(a c-b^{2} \geq \delta>0\right)$, but degenerates at $z=0$. Ignoring the $\theta$ dependence of $N$ one can say that the differential equation has an irregular singular point at $z=0$. Near $z=0$ the terms $c \Delta_{\theta} N-k N_{z}$ dominate, and the equation is more parabolic (with $z$ as time variable) than elliptic: the coefficient $k$ is strictly positive. Assuming Hölder continuity of $u$ and $f$ with respect to $\theta$ we will show below that one can get the same Schauder estimates in the angle variable for $N_{z}, N_{\theta}, N_{\theta \theta}$, which one also has for the parabolic equation

$$
c \Delta_{\theta} N-k N_{z}=f,
$$

as well as Schauder estimates for $z^{2} N_{z z}$ and $z N_{z \theta}$.

We will let $L$ act on functions $u: Q \rightarrow R$ where $Q=[-1,0) \times \mathbb{R}^{d-1}$. For such a function we introduce the Hölder type norm

$$
\operatorname{lip}_{0, \alpha}(u)=\sup \left\{\frac{\left|u(x, \theta)-u\left(x, \theta^{\prime}\right)\right|}{\left|\theta-\theta^{\prime}\right|^{\alpha}}:-1 \leq x<0, \theta \neq \theta^{\prime}\right\} .
$$


The main a priori estimate we derive is:

Theorem 5.1. If $u \in C^{2}(Q)$ satisfies $L u=f$ and if $u(-1, \theta)=0$ for all $\theta \in \mathbb{R}^{d-1}$, then

$$
\operatorname{lip}_{0, \alpha}\left(u_{z}\right)+\operatorname{lip}_{0, \alpha}\left(z^{2} u_{z z}\right)+\operatorname{lip}_{0, \alpha}\left(z u_{z \theta}\right)+\operatorname{lip}_{0, \alpha}\left(u_{\theta_{i} \theta_{j}}\right) \leq C\left(\operatorname{lip}_{0, \alpha}(f)+\sup _{Q}|f|\right) .
$$

Define $h^{0, \alpha}(Q)$ to be the completion of $C^{\infty}(Q)$ under the norm

$$
\|u\|_{h^{0, \alpha}}=\sup _{Q}|u|+\operatorname{lip}_{0, \alpha}(u) .
$$

Within this space we consider the closed subspace

$$
h_{0}^{0, \alpha}(Q)=\left\{u \in h^{0, \alpha}(Q): u(-1, \theta) \equiv 0 \text { for all } \theta \in \mathbb{R}^{d-1}\right\} .
$$

For any $\theta_{0} \in \mathbb{R}^{d-1}$ we define the translation operator

$$
\mathrm{T}_{\theta_{0}} u(\theta)=u\left(\theta+\theta_{0}\right) .
$$

The heat semigroup is given by

$$
e^{t \Delta_{\theta}}=(4 \pi t)^{-(d-1) / 2} \int_{\mathbb{R}^{d-1}} e^{-\theta^{2} / 4 \pi t} \mathrm{~T}_{\theta} d \theta
$$

The Hölder norms can be expressed in terms of the translation group by

$$
\operatorname{lip}_{0, \alpha}(u)=\sup _{\theta \neq 0}|\theta|^{-\alpha}\left\|\mathrm{T}_{\theta} u-u\right\|_{\infty}
$$

where

$$
\|u\|_{\infty}=\sup _{Q}|u|
$$

Lemma 5.2. The operator $L$ is the generator of a strongly continuous contraction semigroup on $h_{0}^{0, \alpha}(Q)$ which commutes with the translations $\left\{\mathrm{T}_{\theta_{0}}: \theta_{0} \in \mathbb{R}^{d-1}\right\}$, and hence with the heat semigroup $e^{t \Delta_{\theta}}$.

Proof. We first show that for any $\lambda>0$ and any bounded $f \in C^{\infty}(Q)$ the equation

$$
L u-\lambda u=f
$$

has a unique bounded classical solution. This is easy: the equation is elliptic in the interior and $\pm \frac{1}{\lambda} \sup _{Q}|f|$ provide sub- and super solutions so that Perron's method gives us existence of at least one bounded solution $u \in C^{2}(Q)$.

To prove uniqueness of the solution we let $m(z)$ be the solution of the ODE

$$
\begin{aligned}
& a z^{2} m^{\prime \prime}(z)-k m^{\prime}(z)=0, \\
& m(-1)=0, \quad m^{\prime}(-1)=1
\end{aligned}
$$

on the interval $-1 \leq z<0$. One finds that $m^{\prime}(z)=C e^{-a / k z}$ so that $m(z)$ grows faster than any negative power $|z|^{-l}$ as $z \nearrow 0$. The function $m$ satisfies $L m=0$.

If there were two bounded solutions to $L u-\lambda u=f$, then their difference $v$ would satisfy $L v-\lambda v=0$. Since $v$ is bounded, $v-\varepsilon m$ attains a local maximum, and $L(v-\varepsilon m)=\lambda v$ implies that this maximum cannot be positive. Thus $v \leq \varepsilon m$ for all $\varepsilon>0$, i.e. $v \leq 0$. The same argument applied to $-v$ implies $v \equiv 0$.

Thus we have shown that $(L-\lambda)^{-1}$ is bounded on $C_{0}(Q) \cap C^{\infty}(Q)$ with norm $\leq \lambda^{-1}$. Since smooth functions are dense in $C_{0}(Q)$ we can extend the resolvent $(L-\lambda)^{-1}$ to all of $C_{0}(Q)$. The Hille-Yosida theorem tells us that $L$ generates a contraction semigroup on $C(Q)$, given by, e.g., $e^{t L}=\lim _{n \rightarrow \infty}\left(I-\frac{t}{n} L\right)^{-n}$. 
Clearly the resolvent and semigroup commute with translations in the $\theta$ direction. This implies that $e^{t L}$ is also a contraction semigroup on the Hölder space $h_{0}^{0, \alpha}(Q)$.

5.1. The $A+B$ lemma. To estimate $u_{\theta \theta}$ in terms of $L u$ we use the following abstract version of a Schauder estimate. Let $e^{t A}$ and $e^{t B}$ be two strongly continuous semigroups on a Banach space $X$, and assume $e^{t A}$ is an analytic semigroup, both $e^{t A}$ and $e^{t B}$ decay exponentially,

$$
\left\|e^{t A}\right\|+\left\|e^{t B}\right\| \leq C e^{-\delta t}
$$

and, above all, assume that the two semigroups commute:

$$
e^{t B} e^{t A}=e^{t A} e^{t B}
$$

Then the family of operators $\mathrm{U}(t)=e^{t A} e^{t B}$ is a $\left(C_{0}\right)$ semigroup. It is thus of the form $\mathrm{U}(t)=e^{t C}$ for some generator $C$. One finds that $D(C) \supset D(A) \cap D(B)$, and that for all $u \in D(A) \cap D(B)$ one has $(A+B) u=C u$, so in a sense one can consider the operator $C$ to be the closed sum of $A+B$. In general the operator $C$ has a strictly larger domain than $D(A) \cap D(B)$, so that one does not expect the operator $A(A+B)^{-1}$ to be bounded, or even well defined. However, consider the interpolation space

$$
D_{\beta, \infty}(A)=\left\{u \in X: \sup _{t>0} t^{-\beta}\left\|A(t-A)^{-1} u\right\|<\infty\right\}
$$

with obvious norm $\|u\|_{\beta, \infty}=\sup _{t>0} t^{-\beta}\left\|A(t-A)^{-1} u\right\|$. Then one can show

Lemma 5.3. For any $u \in D_{\beta, \infty}(A)$ one has $C^{-1} u \in D(A) \cap D(B)$. Moreover there exists a constant $C(\beta)<\infty$ such that

$$
\left\|A C^{-1} u\right\|_{\beta, \infty}+\left\|B C^{-1} u\right\|_{\beta, \infty} \leq C(\beta)\|u\|_{\beta, \infty} .
$$

Since $C$ and $A+B$ coincide on $D(A) \cap D(B)$, this justifies the notation $C^{-1}=$ $(A+B)^{-1}$.

For a proof of this lemma see [21].

In our context we can take

$$
A=\varepsilon \Delta_{\theta}-\frac{1}{2} \quad \text { and } \quad B=a z^{2} \partial_{z}^{2}+2 b z \partial_{z} \partial_{\theta}+(c-\varepsilon) \Delta_{\theta}-k \partial_{z}-\frac{1}{2},
$$

where we take $\varepsilon>0$ so small that $a(c-\varepsilon)-b^{2}>0$ holds, i.e. so that Lemma 5.2 applies to $B$. The constants $\frac{1}{2}$ guarantee that the semigroups decay exponentially.

The interpolation space $D_{\beta, \infty}(A)$ is known to be a "little Hölder space"

$$
D_{\beta, \infty}(A)=h^{0, \alpha+2 \beta}(Q)
$$

provided $0<\alpha+2 \beta<1$. (See [9].)

The semigroups generated by $A$ and $B$ clearly commute, so the lemma implies that the $h^{0, \alpha+\beta}$ norm of $\Delta_{\theta} u$ is bounded by $(A+B) u=L u-u$. Since we can prescribe $0<\alpha+\beta<1$ arbitrarily we have the following a priori estimate:

$$
\left\|\Delta_{\theta} u\right\|_{h^{0, \alpha}} \leq C_{\alpha}\|L u-u\|_{h^{0, \alpha}} .
$$


5.2. Estimation of $\partial_{z} u$. Using the maximum principle we can extract first an $L^{\infty}$ bound for $\partial_{z} u$ from (5.1) and then, using translation invariance, an $h^{0, \alpha}$ bound.

Lemma 5.4. Let $u \in C^{2}(Q)$ be a function for which both Lu and $\Delta_{\theta} u$ are uniformly bounded, and for which $u(-1, \theta) \equiv 0$. Then $\partial_{z} u$ is also uniformly bounded, in fact $\left\|\partial_{z} u\right\|_{\infty} \leq C\left(\|L u\|_{\infty}+\left\|\Delta_{\theta} u\right\|_{\infty}\right)$ for some constant $C<\infty$.

Proof. Since $L(z+1)=-k$, the maximum principle implies that $\|u\|_{\infty} \leq k^{-1}\|L u\|_{\infty}$, so $u$ is also uniformly bounded, and by interpolation with the $\left\|\Delta_{\theta} u\right\|_{\infty}$ estimate, $\nabla_{\theta} u$ is also uniformly bounded.

Let $\left(z_{0}, \theta_{0}\right)$ with $-1 \leq z<0$ be given. Then

$$
v_{ \pm}(z, \theta)=u\left(z_{0}, \theta\right) \pm K\left(z-z_{0}\right)
$$

satisfies $L v_{ \pm}=c \Delta_{\theta} u\left(z_{0}, \theta\right) \mp k K$, so they are sub- and super solutions on $\left[z_{0}, 0\right) \times$ $\mathbb{T}^{d-1}$ provided $K$ is chosen larger than $k^{-1}\left(c\left\|\Delta_{\theta} u\right\|_{\infty}+\|L u\|_{\infty}\right)$. Thus for $z_{0} \leq$ $z<0$ one has $v_{-} \leq u \leq v_{+}$which implies at $z=z_{0}$ that

$$
\left|u_{z}\left(z_{0}, \theta\right)\right| \leq K=\frac{1}{k}\left(c\left\|\Delta_{\theta} u\right\|_{\infty}+\|L u\|_{\infty}\right) .
$$

Lemma 5.5. For $u \in h^{0, \alpha}(Q)$ with $u(-1, \theta) \equiv 0$ and $L u \in h^{0, \alpha}(Q)$ one has

$$
\left\|\partial_{z} u\right\|_{h^{0, \alpha}} \leq \frac{1}{k}\left(c\left\|\Delta_{\theta} u\right\|_{h^{0, \alpha}}+\|L u-u\|_{h^{0, \alpha}}\right) \leq C\|L u-u\|_{h^{0, \alpha}} .
$$

Proof. From (5.1) we see that $\Delta_{\theta} u \in h^{0, \alpha}(Q)$. The function $v_{\sigma}(z, \theta)=u(z, \theta+\sigma)-$ $u(z, \theta)$ satisfies

$$
\left\|\Delta_{\theta} v_{\sigma}\right\|_{\infty} \leq\left\|\Delta_{\theta} u\right\|_{h^{0, \alpha}}|\sigma|^{\alpha} \quad \text { and } \quad\left\|L v_{\sigma}\right\|_{\infty} \leq\|L u\|_{h^{0, \alpha}}|\sigma|^{\alpha},
$$

so the previous lemma gives us

$$
\left\|\partial_{z} v_{\sigma}\right\|_{\infty} \leq \frac{1}{k}\left(c\left\|\Delta_{\theta} u\right\|_{h^{0, \alpha}}+\|L u\|_{h^{0, \alpha}}\right)|\sigma|^{\alpha},
$$

which implies the stated estimate.

5.3. Estimation of the other terms in $L u$. We have found that $L u \in h^{0, \alpha}(Q)$ implies that both $\Delta_{\theta} u$ and $u_{z}$ belong to $h^{0, \alpha}(Q)$. Classical Schauder estimates for the Laplacian imply that all second derivatives $\partial_{\theta_{i}} \partial_{\theta_{j}} u$ also belong to $h^{0, \alpha}(Q)$. Thus the remaining terms, i.e.

$$
L u+k u_{z}=a z^{2} u_{z z}+2 b z u_{z \theta}+c \Delta_{\theta} u,
$$

also belong to $h^{0, \alpha}(Q)$. If we now change the $z$ variable to $\xi=\ln |z|$, then we find that

$$
L u+(k+a z) u_{z}=a u_{\xi \xi}+2 b u_{\xi \theta}+c \Delta_{\theta} u
$$

is defined for all $\xi \geq 0$ and uniformly $\alpha$-Hölder continuous in the $\theta$ variable on $\left\{(\xi, \theta): \xi \geq 0, \theta \in \mathbb{R}^{\bar{d}-1}\right\}$. The right-hand side is a uniformly elliptic constant coefficient operator, so classical Schauder estimates once again imply that the individual terms $u_{\xi \xi}$ and $u_{\xi \theta}$ are uniformly $\alpha$-Hölder continuous in $\theta$. Translated back to the $z$ variable this implies that $z^{2} u_{z z}$ and $z u_{z \theta}$ both belong to $h^{0, \alpha}(Q)$. One also gets the corresponding a priori estimates so that we have completed the proof of Theorem 5.1 . 
5.4. Variable coefficients. Let $L$ be the differential operator

$$
L=a z^{2} \partial_{z}^{2}+2 b z \partial_{z} \partial_{\theta}+c \Delta_{\theta}-k \partial_{z},
$$

where $a, b, c$, and $k$ are now functions in $h^{0, \alpha}(Q)$ which satisfy

$$
a c-b^{2} \geq \delta>0 \quad \text { and } \quad k \geq \delta>0 .
$$

Theorem 5.1 provides Schauder estimates for this operator in the case that the coefficients $a, b, c$, and $k$ are constant. By "freezing the coefficients" we deduce from this the following:

Theorem 5.6. If $u \in C^{2}(Q)$ satisfies $L u=f$ and if $u(-1, \theta)=0$ for all $\theta \in \mathbb{R}^{d-1}$, then

$\operatorname{lip}_{0, \alpha}\left(u_{z}\right)+\operatorname{lip}_{0, \alpha}\left(z^{2} u_{z z}\right)+\operatorname{lip}_{0, \alpha}\left(z u_{z \theta}\right)+\operatorname{lip}_{0, \alpha}\left(u_{\theta_{i} \theta_{j}}\right) \leq C\left(\operatorname{lip}_{0, \alpha}(f)+\sup _{Q}|f|\right)$.

In the above we may replace $\mathbb{R}^{d-1}$ by any other $d-1$ dimensional manifold, such as $S^{d-1}$, and $Q$ by $Q=[-1,0] \times S^{d-1}$ and obtain the same estimates.

\section{Schauder estimates at $z=-1$}

Near $z=-1$ or, equivalently, $\eta=0$, we must find Schauder estimates for the operator

$$
M=\eta\left(\partial_{\eta}^{2}+\partial_{\theta}^{2}\right)+a \partial_{\eta}+b_{j} \partial_{\theta_{j}} .
$$

We will obtain such estimates in the space $h^{0, \alpha}(Q)$, where now $Q=(0, \infty) \times \mathbb{R}^{d-1}$. Our method here will be to use the Fourier transform in the $\theta$ variables. The key to proving Schauder estimates via the Fourier transform is presented in Section VI.5.3 of 22] (see also the section on Schauder estimates for constant coefficient elliptic operators in [19]). We first outline the formal solution, and then present the estimates.

To solve

$$
M u=f
$$

on $Q$ we let $U(\eta, \lambda)$ be the Fourier transform of $u$ with respect to $\theta, \lambda_{j}$ being the dual variable to $\theta_{j}$, i.e.

$$
U(\eta, \lambda)=(2 \pi)^{-(d-1) / 2} \int_{\mathbb{R}^{d-1}} e^{-i \lambda \theta} u(\eta, \theta) d \theta .
$$

The transform then satisfies the equation

$$
\eta U^{\prime \prime}+a U^{\prime}+\left(i b_{j} \lambda_{j}-\eta \lambda^{2}\right) U=F,
$$

where $F$ is the transform of $f$. This ODE has exactly one solution which is regular at both $\eta=0$ and $\eta=\infty$. That solution is given by the variation of constants formula

$$
U(\eta, \lambda)=\int_{0}^{\infty} K\left(i b_{j} \lambda_{j}, \lambda^{2} ; \eta, \eta^{\prime}\right) F\left(\eta^{\prime}, \lambda\right) d \eta^{\prime}
$$

where

$$
K\left(p, q ; \eta, \eta^{\prime}\right)=\left\{\begin{array}{l}
\frac{\phi_{0}(\eta) \phi_{\infty}\left(\eta^{\prime}\right)}{\eta^{\prime} W\left(\eta^{\prime}\right)} \text { for } \eta<\eta^{\prime} \\
\frac{\phi_{0}\left(\eta^{\prime}\right) \phi_{\infty}(\eta)}{\eta^{\prime} W\left(\eta^{\prime}\right)} \text { for } \eta>\eta^{\prime}
\end{array}\right.
$$


and $\phi_{0, \infty}(\eta)$ are solutions of

$$
\eta \phi^{\prime \prime}+a \phi^{\prime}+(p-q \eta) \phi=0
$$

with boundary conditions:

$$
\left.\phi_{0}(\eta)=1+\sum_{j=1}^{\infty} c_{j} \eta^{j} \quad \text { (i.e. } \phi_{0} \text { is analytic around } \eta=0\right)
$$

and

$$
\phi_{\infty}(\eta)=\eta^{\frac{a}{2}+\frac{p}{2 \sqrt{q}}} e^{-\sqrt{q} \eta}(1+o(1)) \quad(\eta \rightarrow \infty) .
$$

Here $W(\eta)=\phi_{0}(\eta) \phi_{\infty}^{\prime}(\eta)-\phi_{0}^{\prime}(\eta) \phi_{\infty}(\eta)$ is the Wronskian of these two functions.

Taking the inverse Fourier transform of the kernel $K$,

$$
\check{K}\left(\theta ; \eta, \eta^{\prime}\right)=(2 \pi)^{-(d-1) / 2} \int_{\mathbb{R}^{d-1}} e^{i \lambda \theta} K\left(i b \lambda, \lambda^{2} ; \eta, \eta^{\prime}\right) d \lambda,
$$

we can then write the solution $u$ of $M u=f$ as

$$
u(\eta, \theta)=\int_{0}^{\infty} \check{K}\left(\theta ; \eta, \eta^{\prime}\right) * f\left(\eta^{\prime}, \theta\right) d \eta^{\prime},
$$

where $*$ stands for convolution in the $\theta$ variables. A dyadic decomposition of the kernel $K$ in Fourier space will lead to a proof of:

Theorem 6.1. Let $u \in C^{\infty}\left([0, \infty) \times \mathbb{R}^{d-1}\right)$ be a compactly supported function. Then

$$
\left\|\eta u_{\eta \eta}\right\|_{h^{0, \alpha}}+\left\|u_{\eta}\right\|_{h^{0, \alpha}}+\left\|u_{\theta_{i}}\right\|_{h^{0, \alpha}}+\left\|\eta u_{\theta_{i} \theta_{j}}\right\|_{h^{0, \alpha}} \leq C\left\{\|u\|_{h^{0, \alpha}}+\|M u\|_{h^{0, \alpha}}\right\}
$$

for some constant $C=C(\alpha, a, d)$.

6.1. Green's function. Consider the unbounded operator $L: D(L) \rightarrow L^{\infty}\left(\mathbb{R}_{+}\right)$ given by

$$
\begin{aligned}
L u & =\eta u^{\prime \prime}+a u^{\prime}+(p-q \eta) u \\
D(L) & =\left\{u \in L^{\infty}\left(\mathbb{R}_{+}\right): \eta u^{\prime \prime}, u^{\prime}, \eta u \in L^{\infty}\left(\mathbb{R}_{+}\right)\right\} .
\end{aligned}
$$

The operator depends on parameters $a, p, q$, while its domain does not. We give this domain the norm

$$
\|u\|_{D(L)}=\left\|\eta u^{\prime \prime}\right\|_{\infty}+\left\|u^{\prime}\right\|_{\infty}+\|(1+\eta) u\|_{\infty}
$$

with which it becomes a Banach space.

Lemma 6.2. For all $u \in D(L)$ one has $\eta u^{\prime} \in L^{\infty}\left(\mathbb{R}_{+}\right)$, and $\left\|\eta u^{\prime}\right\|_{\infty} \leq C\|u\|_{D(L)}$ for some finite constant $C$.

Proof. This follows from the calculus inequality

$$
\sup _{a \leq x \leq b}\left|u^{\prime}(x)\right| \leq(b-a) \sup _{a \leq x \leq b}\left|u^{\prime \prime}(x)\right|+\frac{1}{b-a} \underset{a \leq x \leq b}{\operatorname{osc}}|u(x)|
$$

applied to the interval $[a, b]=[\eta, \eta+1]$.

Lemma 6.3. $L$ is invertible for all $a>0, p \in \mathbb{C}$, and $q \in \mathbb{C} \backslash(-\infty, 0]$. 
Proof. For any given $f \in L^{\infty}$ one can use ODE arguments to construct two solutions $u_{0}, u_{\infty}$ of

$$
\eta u^{\prime \prime}+a u^{\prime}+(p-q \eta) u=f,
$$

where $u_{0}$ is regular at $\eta=0$, in the sense that its derivative is bounded there, and where $u_{\infty}$ is regular at $\eta=\infty$, meaning $\eta u$ is bounded. Indeed at $\eta=0$ the equation is a perturbation of $\eta u^{\prime \prime}+a u^{\prime}=f$, and at $\eta=\infty$ the equation is a perturbation of $u^{\prime \prime}-q u=f / \eta$; both of these equations can be solved explicitly, and (Picard) iteration provides $u_{0}, u_{\infty}$.

The difference $v=u_{0}-u_{\infty}$ satisfies the homogeneous equation which is of confluent hypergeometric type. (The substitution $\phi=\eta^{-a / 2} \sigma$ leads to an equation of the form $\sigma^{\prime \prime}-\left\{A / \eta^{2}+B / \eta+C\right\} \sigma=0$ whose solution can be written in terms of Whittaker functions $W_{k . m}(2 \sqrt{C} \eta)$. See [23], but we will not use the "explicit" form of the solution here.) The restrictions on the parameters $a, p, q$ imply that the homogeneous equation has two linearly independent solutions $\phi_{0}, \phi_{\infty}$ as described above with $\phi_{0}$ regular at $\eta=0$ and $\phi_{\infty}$ regular at $\eta=\infty$. Then $v$ is of the form $v=c_{0} \phi_{0}+c_{\infty} \phi_{\infty}$ and it follows that

$$
u=u_{0}-c_{0} \phi_{0}=u_{\infty}+c_{\infty} \phi_{\infty}
$$

is a solution to $L u=f$ which satisfies the boundary conditions, i.e. which belongs to $D(L)$.

Since $\phi_{0}$ is unbounded at $\eta=\infty$ and $\phi_{\infty}$ has unbounded derivative at $\eta=0$, there is no solution $v \in D(L)$ of $L v=0$, and thus the solution $u \in D(L)$ of $L u=f$ is unique.

Since the operator $L: D(L) \rightarrow L^{\infty}\left(\mathbb{R}_{+}\right)$is bounded, the closed graph theorem implies that its inverse is also bounded. The inverse is an integral operator whose kernel is, of course, Green's function. Boundedness of $(1+\eta) L^{-1},(1+\eta) \partial_{\eta} \circ L^{-1}$, and $\eta \partial_{\eta}^{2} \circ L^{-1}$ on $L^{\infty}$ implies the following estimates for Green's function:

Lemma 6.4. For any $a>0, p \in \mathbb{C}$, and $q \in \mathbb{C} \backslash(-\infty, 0]$ one has

$$
\begin{aligned}
& (1+\eta) \int_{0}^{\infty}\left|K\left(p, q ; \eta, \eta^{\prime}\right)\right| d \eta^{\prime} \leq A, \\
& (1+\eta) \int_{0}^{\infty}\left|K_{\eta}\left(p, q ; \eta, \eta^{\prime}\right)\right| d \eta^{\prime} \leq A
\end{aligned}
$$

uniformly in $\eta>0$. The constants $A$ depend continuously on the parameters $a, p, q$.

The inverse function theorem says that $L^{-1}$ depends analytically on any parameters which $L$ happens to contain, so the map $(a, p, q) \longmapsto L^{-1}$ is smooth. This implies that the kernel $K$ depends smoothly on $p$ and $q$ and that the derivatives satisfy the same estimates as (6.2) and 6.3 :

Lemma 6.5. For any $a>0, p \in \mathbb{C}$, and $q \in \mathbb{C} \backslash(-\infty, 0]$ one has

$$
\begin{aligned}
& (1+\eta) \int_{0}^{\infty}\left|\partial_{p}^{k} \partial_{q}^{l} K\left(p, q ; \eta, \eta^{\prime}\right)\right| d \eta^{\prime} \leq A_{k, l}, \\
& (1+\eta) \int_{0}^{\infty}\left|\partial_{p}^{k} \partial_{q}^{l} K_{\eta}\left(p, q ; \eta, \eta^{\prime}\right)\right| d \eta^{\prime} \leq A_{k, l}
\end{aligned}
$$

uniformly in $\eta>0$. The constants $A_{k, l}$ again depend continuously on the parameters $a, p, q$. 
Here we have obtained (6.2) $-(6.5)$ by functional analytic arguments (i.e. the closed graph theorem combined with suppression of some details such as Picard iteration); one could also derive these estimates by a careful analysis of the asymptotics of the solutions $\phi_{0}$ and $\phi_{\infty}$ at $\eta=0$ and $\eta=\infty$ (e.g. by using their representation as Whittaker functions and citation of classical results on these functions) followed by a direct estimation of the relevant integrals. Either way, we leave the tedious details to the reader.

If one substitutes $u(\eta)=v(\eta / r)$ in the ODE $L u=f$, then one gets, with $\xi=\eta / r$,

$$
\eta u^{\prime \prime}+a u^{\prime}+(p-q \eta) u=f(\eta) \Longrightarrow \xi v^{\prime \prime}+a v^{\prime}+\left(r p-r^{2} q \xi\right) v=r f(r \xi) .
$$

This leads to the following homogeneity property of the kernel $K$ :

Lemma 6.6. For any $r>0$ one has

$$
K\left(\frac{p}{r}, \frac{q}{r^{2}} ; \eta, \eta^{\prime}\right)=K\left(p, q ; \frac{\eta}{r}, \frac{\eta^{\prime}}{r}\right) .
$$

6.2. An equivalent norm on Hölder spaces. Let $\psi \in \mathcal{S}\left(\mathbb{R}^{d-1}\right)$ be a function whose Fourier transform $\hat{\psi}$ has compact support and satisfies

$$
\hat{\psi}(\lambda)=\left\{\begin{array}{l}
1 \text { for }|\lambda| \leq 1 \\
0 \text { for }|\lambda| \geq 2
\end{array}\right.
$$

We define $\chi_{j} \in \mathcal{S}\left(\mathbb{R}^{d-1}\right)$ by $\hat{\chi}(\lambda)=\hat{\psi}(\lambda / 2)-\hat{\psi}(\lambda)$, and

$$
\hat{\chi}_{j}(\lambda)=\hat{\chi}\left(2^{-j} \lambda\right), \quad \text { i.e. } \chi_{j}(\theta)=2^{j(d-1)} \chi\left(2^{j} \theta\right) .
$$

Then one has

$$
\hat{\psi}(\lambda)+\sum_{j=0}^{\infty} \hat{\chi}_{j}(\lambda)=1
$$

and

$$
\operatorname{supp} \hat{\chi}_{j} \subset B\left(0 ; 2^{j+1}\right) \backslash B\left(0 ; 2^{j-1}\right)
$$

which implies

$$
\left(\chi_{j-1}+\chi_{j}+\chi_{j+1}\right) * \chi_{j}=\chi_{j}
$$

The function $\chi_{j}$ decays faster than any power of $|\theta|^{-1}$ as $\theta \rightarrow \infty$, and all its moments vanish (since its Fourier transform vanishes in a neighborhood of $\lambda=0)$. From this one can show that $u \in C^{m+\alpha}\left(\mathbb{R}^{d-1}\right)$ implies $\left\|\chi_{j} * u\right\|_{\infty} \leq$ $C 2^{-j(m+\alpha)} \operatorname{lip}_{\alpha}\left(D^{m} u\right)$. In fact it is known that this inequality is sharp, and that the quantity

$$
[u]_{m+\alpha} \stackrel{\text { def }}{=}\|\psi * u\|_{\infty}+\sup _{j \geq 0} 2^{j(m+\alpha)}\left\|\chi_{j} * u\right\|_{\infty}
$$

defines a norm which is equivalent to the usual Hölder norm on $C^{m+\alpha}\left(\mathbb{R}^{d-1}\right)$, provided $m \geq 0$ and $0<\alpha<1$. (See [22].) 
6.3. The kernel decomposed. For a fixed vector $b \in \mathbb{R}^{d-1}$ we define

$$
k\left(\lambda ; \eta, \eta^{\prime}\right)=K\left(i b_{j} \lambda_{j}, \lambda_{1}^{2}+\cdots+\lambda_{d-1}^{2} ; \eta, \eta^{\prime}\right)
$$

and for $j \geq 0$,

$$
k_{j}\left(\lambda ; \eta, \eta^{\prime}\right)=\left(\hat{\chi}_{j-1}(\lambda)+\hat{\chi}_{j}(\lambda)+\hat{\chi}_{j+1}(\lambda)\right) K\left(i b_{j} \lambda_{j}, \lambda_{1}^{2}+\cdots+\lambda_{d-1}^{2} ; \eta, \eta^{\prime}\right) .
$$

According to Lemma 6.5 $k_{j}$ is a smooth function of $\lambda$ for each $\eta, \eta^{\prime}$ and by construction it is supported in the shell $2^{j-2} \leq|\lambda| \leq 2^{j+2}$. Its inverse Fourier transform is therefore a smooth rapidly decaying function and, in particular, $\breve{k}_{j}\left(\theta ; \eta, \eta^{\prime}\right) \in$ $L^{1}\left(\mathbb{R}^{d-1}\right)$ for any $\eta, \eta^{\prime}>0$. Lemma 6.5 also implies that

$$
\begin{gathered}
(1+\eta) \int_{0}^{\infty}\left\|\check{k}_{j}\left(\theta ; \eta, \eta^{\prime}\right)\right\|_{L^{1}\left(\mathbb{R}^{d-1}\right)} d \eta^{\prime} \leq A_{j}, \\
(1+\eta) \int_{0}^{\infty}\left\|\partial_{\eta} \check{k}_{j}\left(\theta ; \eta, \eta^{\prime}\right)\right\|_{L^{1}\left(\mathbb{R}^{d-1}\right)} d \eta^{\prime} \leq A_{j}
\end{gathered}
$$

for certain constants $A_{j}$.

We now use the homogeneity in Lemma 6.6 to derive the dependence of the constants $A_{j}$ on $j$. Write $\chi_{-1 ;+1}$ for $\chi_{-1}+\chi_{0}+\chi_{1}$. Lemma 6.6 implies that

$$
\begin{aligned}
k_{j}\left(\lambda ; \eta, \eta^{\prime}\right) & =\hat{\chi}_{-1 ;+1}\left(2^{-j} \lambda\right) K\left(i b \lambda, \lambda^{2} ; \eta, \eta^{\prime}\right) \\
& =\hat{\chi}_{-1 ;+1}\left(2^{-j} \lambda\right) K\left(i b 2^{-j} \lambda, 2^{-2 j} \lambda^{2} ; 2^{j} \eta, 2^{j} \eta^{\prime}\right) \\
& =k_{0}\left(2^{-j} \lambda ; 2^{j} \eta, 2^{j} \eta^{\prime}\right)
\end{aligned}
$$

so that the Fourier transforms satisfy

$$
\check{k}_{j}\left(\theta ; \eta, \eta^{\prime}\right)=2^{j(d-1)} \check{k}_{0}\left(2^{j} \theta ; 2^{j} \eta, 2^{j} \eta^{\prime}\right) .
$$

For fixed $\eta, \eta^{\prime}>0$ this shows that $\check{k}_{j}\left(\theta ; \eta, \eta^{\prime}\right)$ and $\check{k}_{0}\left(\theta ; 2^{j} \eta, 2^{j} \eta^{\prime}\right)$ have the same $L^{1}\left(\mathbb{R}^{d-1}\right)$ norm. Integration in $\eta^{\prime}$ then gives us:

$$
\begin{aligned}
\int_{0}^{\infty}\left\|\check{k}_{j}\left(\theta ; \eta, \eta^{\prime}\right)\right\|_{L^{1}\left(\mathbb{R}^{d-1}\right)} d \eta^{\prime} & =\int_{0}^{\infty}\left\|\check{k}_{0}\left(\theta ; 2^{j} \eta, 2^{j} \eta^{\prime}\right)\right\|_{L^{1}\left(\mathbb{R}^{d-1}\right)} d \eta^{\prime} \\
& =2^{-j} \int_{0}^{\infty}\left\|\check{k}_{0}\left(\theta ; 2^{j} \eta, \xi\right)\right\|_{L^{1}\left(\mathbb{R}^{d-1}\right)} d \xi \\
& \leq 2^{-j} A_{0} .
\end{aligned}
$$

A similar computation also gives

$$
\begin{gathered}
\eta \int_{0}^{\infty}\left\|\check{k}_{j}\left(\theta ; \eta, \eta^{\prime}\right)\right\|_{L^{1}\left(\mathbb{R}^{d-1}\right)} d \eta^{\prime} \leq 2^{-2 j} A_{0}, \\
\eta \int_{0}^{\infty}\left\|\partial_{\eta} \check{k}_{j}\left(\theta ; \eta, \eta^{\prime}\right)\right\|_{L^{1}\left(\mathbb{R}^{d-1}\right)} d \eta^{\prime} \leq 2^{-j} A_{0},
\end{gathered}
$$

and

$$
\int_{0}^{\infty}\left\|\partial_{\eta} \breve{k}_{j}\left(\theta ; \eta, \eta^{\prime}\right)\right\|_{L^{1}\left(\mathbb{R}^{d-1}\right)} d \eta^{\prime} \leq A_{0} .
$$


6.4. A priori estimates. In this section we prove the main estimate for the operator $M$. As before we define $Q=[0, \infty) \times \mathbb{R}^{d-1}$.

Theorem 6.7. Let $u \in C^{\infty}(Q)$ be compactly supported, with $u(\eta, \theta) \equiv 0$ whenever $|\theta| \geq 1$ or $\eta \geq 1$. Then one has

$$
\begin{array}{r}
\left\|\eta u_{\eta \eta}\right\|_{h^{0, \alpha}}+\left\|\eta u_{\theta_{i} \eta}\right\|_{h^{0, \alpha}}+\left\|\eta u_{\theta_{i} \theta_{j}}\right\|_{h^{0, \alpha}}+\left\|u_{\eta}\right\|_{h^{0, \alpha}}+\left\|u_{\theta_{i}}\right\|_{h^{0, \alpha}} \\
\leq C\left\{\|u\|_{h^{0, \alpha}}+\|M u\|_{h^{0, \alpha}}\right\}
\end{array}
$$

for some constant $C$ which does not depend on $u$.

To prove this we split $u$ into two parts,

$$
u=v+w, \quad v=\psi * u, w=u-\psi * u,
$$

and show that both $v$ and $w$ satisfy the same a priori estimates which we claim for $u$.

Estimating the smooth part $v$. Since the convolution is taken in the $\theta$ variables only, we know that $v$ vanishes for $\eta \geq 1$, and thus we can estimate $\left\|\eta v_{\theta_{i} \theta_{j}}\right\|_{h^{0, \alpha}}$ and $\left\|v_{\theta_{i}}\right\|_{h^{0, \alpha}}$ via

$$
\left\|v_{\theta_{i}}\right\|_{h^{0, \alpha}}=\left\|\psi_{\theta_{i}} * u\right\|_{h^{0, \alpha}} \leq\left\|\psi_{\theta_{i}}\right\|_{L^{1}\left(\mathbb{R}^{d-1}\right)}\|u\|_{h^{0, \alpha}}
$$

and

$$
\left\|v_{\theta_{j} \theta_{i}}\right\|_{h^{0, \alpha}}=\left\|\psi_{\theta_{j} \theta_{i}} * u\right\|_{h^{0, \alpha}} \leq\left\|\psi_{\theta_{j} \theta_{i}}\right\|_{L^{1}\left(\mathbb{R}^{d-1}\right)}\|u\|_{h^{0, \alpha}}
$$

in terms of $\|u\|_{h^{0, \alpha}}$. We then have

$$
\eta v_{\eta \eta}+a v_{\eta}=M v-b_{j} v_{\theta_{j}}-\eta \Delta_{\theta} v \stackrel{\text { def }}{=} g(\eta, \theta),
$$

where the right-hand side is bounded in $h^{0, \alpha}$ in terms of $\|u\|_{h^{0, \alpha}}$ and $\|M u\|_{h^{0, \alpha}}$ (use $M v=\psi * M u)$.

Integration gives

$$
v_{\eta}=\int_{0}^{\eta}\left(\frac{\eta^{\prime}}{\eta}\right)^{a} g\left(\eta^{\prime}, \theta\right) \frac{d \eta^{\prime}}{\eta^{\prime}}=\int_{0}^{1} \tau^{a-1} g(\tau \eta, \theta) d \tau
$$

which shows that $\left\|v_{\eta}\right\|_{h^{0, \alpha}}$ is bounded by $C\|g\|_{h^{0, \alpha}}$ and hence by

$$
C\left\{\|u\|_{h^{0, \alpha}}+\|M u\|_{h^{0, \alpha}}\right\} .
$$

Since $\eta v_{\eta \eta}=g-a v_{\eta}$, we also get a bound for $\left\|\eta v_{\eta \eta}\right\|_{h^{0, \alpha}}$.

Finally, to estimate $\left\|\eta v_{\theta_{i} \eta}\right\|_{h^{0, \alpha}}$ we consider $\zeta(\theta) \stackrel{\text { def }}{=} \psi(2 \theta)$. Then $\hat{\zeta}(\lambda)=\hat{\psi}(\lambda / 2)$ so $\hat{\zeta}(\lambda)=1$ on the support of $\hat{\psi}(\lambda)$, and we have $\zeta * \psi=\psi$. Hence

$$
v_{\theta_{i} \eta}=\partial_{\theta_{i}}\left(\psi * \partial_{\eta} u\right)=\partial_{\theta_{i}}\left(\zeta * \psi * \partial_{\eta} u\right)=\zeta_{\theta_{i}} * \psi * \partial_{\eta} u=\zeta_{\theta_{i}} * v_{\eta} .
$$

Since $v_{\eta}$ is bounded in $h^{0, \alpha}$, we also see that $v_{\theta_{i} \eta}$ and thus $\eta v_{\eta \theta_{i}}$ are bounded in $h^{0, \alpha}$. 
Estimating the non-smooth part $w=u-\psi * u$. Let $f=M w=M u-\psi * M u$. By our equivalent description of the Hölder norm we have

$$
\sup _{\eta>0}\left\|\chi_{j} * f(\eta, \theta)\right\|_{\infty} \leq C 2^{-j \alpha}\|f\|_{h^{0, \alpha}(Q)} \leq C^{\prime} 2^{-j \alpha}\|M u\|_{h^{0, \alpha}(Q)} .
$$

To estimate $w$ and its derivatives in terms of $f$ we begin with

$$
w(\eta, \theta)=\int_{0}^{\infty} \check{k}\left(\theta ; \eta, \eta^{\prime}\right) * f\left(\eta^{\prime}, \theta\right) d \eta^{\prime}
$$

which leads to

$$
\begin{aligned}
\chi_{j} * w & =\int_{0}^{\infty} \chi_{j} * \check{k}\left(\theta ; \eta, \eta^{\prime}\right) * f\left(\eta^{\prime}, \theta\right) d \eta^{\prime} \\
& =\int_{0}^{\infty} \check{k}_{j}\left(\theta ; \eta, \eta^{\prime}\right) *\left\{\chi_{j} * f\left(\eta^{\prime}, \theta\right)\right\} d \eta^{\prime},
\end{aligned}
$$

so that

$$
\begin{aligned}
\left\|\chi_{j} * w\right\|_{\infty} & \leq \int_{0}^{\infty}\left\|\check{k}_{j}\left(\theta ; \eta, \eta^{\prime}\right)\right\|_{L_{1}}\left\|\chi_{j} * f\left(\eta^{\prime}, \theta\right)\right\|_{\infty} d \eta^{\prime} \\
& \leq C 2^{-j \alpha}\|M u\|_{h^{0, \alpha}(Q)} \int_{0}^{\infty}\left\|\check{k}_{j}\left(\theta ; \eta, \eta^{\prime}\right)\right\|_{L_{1}} d \eta^{\prime} \\
& \leq C 2^{-j \alpha}\|M u\|_{h^{0, \alpha}(Q)} \cdot 2^{-2 j} A_{0} \\
& =A_{0} C\|M u\|_{h^{0, \alpha}(Q)} 2^{-j(2+\alpha)}
\end{aligned}
$$

This implies that the $\alpha$ Hölder norms of the $w_{\theta}$ are uniformly bounded:

$$
\left\|w_{\theta}\right\|_{h^{0, \alpha}} \leq C\|M u\|_{h^{0, \alpha}} .
$$

Similar calculations yield

$$
\begin{aligned}
\eta\left\|\chi_{j} * w\right\|_{\infty} & \leq C\|M u\|_{h^{0, \alpha}(Q)} 2^{-j(2+\alpha)}, \\
\left\|\chi_{j} * w_{\eta}\right\|_{\infty} & \leq C\|M u\|_{h^{0, \alpha}(Q)} 2^{-j \alpha}
\end{aligned}
$$

and

$$
\eta\left\|\chi_{j} * w_{\eta}\right\|_{\infty} \leq C\|M u\|_{h^{0, \alpha}(Q)} 2^{-j(1+\alpha)}
$$

which implies

$$
\left\|\eta w_{\theta \theta}\right\|_{h^{0, \alpha}}+\left\|\eta w_{\eta \theta}\right\|_{h^{0, \alpha}}+\left\|w_{\eta}\right\|_{h^{0, \alpha}} \leq C\|M u\|_{h^{0, \alpha}} .
$$

Combining the estimates for $w$ and for $v$ we complete the proof of (6.10).

6.5. Variable coefficients. As before the method of "freezing the coefficients" leads in a completely standard way to a priori estimates for operators with Hölder continuous coefficients. We merely state the result.

Theorem 6.8. Let $Q=[0, \infty) \times S^{d-1}$ and let $\bar{M}$ be the differential operator

$$
\bar{M}=\eta\left\{a_{00}(\eta, \theta) \partial_{\eta}^{2}+2 a_{0 i}(\eta, \theta) \partial_{\eta} \partial_{\theta_{i}}+a_{i j}(\eta, \theta) \partial_{\theta_{i}} \partial_{\theta_{j}}\right\}+b_{0}(\eta, \theta) \partial_{\eta}+b_{i}(\eta, \theta) \partial_{\theta_{i}},
$$

where the coefficients $a_{\lambda \mu}, b_{\nu}$ belong to $h^{0, \alpha}(Q)$ and where

$$
\forall\left(\zeta, \lambda_{i}\right) \in \mathbb{R}^{d}: \delta\left(\zeta^{2}+|\lambda|^{2}\right) \leq a_{00} \zeta^{2}+2 a_{0 i} \zeta \lambda_{i}+a_{i j} \lambda_{i} \lambda_{j} \leq \frac{1}{\delta}\left(\zeta^{2}+|\lambda|^{2}\right)
$$


and

$$
\forall \theta \in S^{d-1}: b_{0}(0, \theta) \geq \delta
$$

hold for some $\delta>0$.

Let $u \in C^{\infty}(Q)$ be compactly supported, with $u(\eta, \theta) \equiv 0$ for all $\eta \geq 1$. Then one has

$$
\begin{aligned}
& \left\|\eta u_{\eta \eta}\right\|_{h^{0, \alpha}}+\left\|\eta u_{\theta_{i} \eta}\right\|_{h^{0, \alpha}}+\left\|\eta u_{\theta_{i} \theta_{j}}\right\|_{h^{0, \alpha}}+\left\|u_{\eta}\right\|_{h^{0, \alpha}}+\left\|u_{\theta_{i}}\right\|_{h^{0, \alpha}} \\
& \quad \leq C\left\{\|u\|_{h^{0, \alpha}}+\|\bar{M} u\|_{h^{0, \alpha}}\right\}
\end{aligned}
$$

for some constant $C$ which does not depend on $u$.

\section{Global Schauder estimates and the Fredholm property}

Let $M$ be the operator of Theorem 4.3. By combining the a priori estimates near $z=0$ and near $z=-1$ which we have obtained in the previous sections we directly get the following estimate: For any $u \in Y$ one has

$$
\|u\|_{Y} \leq C\left\{\|u\|_{X}+\|M u\|_{X}\right\}
$$

for some constant $C<\infty$ which only depends on the operator $M$ and not on $u$. To prove that $M$ is a Fredholm operator we first note that we can drop the lowest order term.

Lemma 7.1. For $\ell \in h^{0, \alpha}\left([-1,0] \times S^{d-1}\right)$ the multiplication operator $u \in Y \mapsto$ $\ell u \in X$ is compact.

Proof. Multiplication with $\ell$ is a bounded operator on $X$ itself, so we only have to show that the inclusion $Y \hookrightarrow X$ is compact. But this follows immediately from the fact that $Y$ is contained in $C^{1}\left([-1,0] \times S^{d-1}\right)$.

Thus we may assume that $\ell(z, \theta)<0$, as we will do in the remainder of this section. In what follows we will have to deal with "strong solutions" and we will need a maximum principle for such solutions. This happens to us because we chose to work in a function space $h^{0, \alpha}$ in which we have no control of the modulus of continuity with respect to the $z$ variable. Fortunately we can quote Gilbarg and Trudinger [15] for all the relevant results.

A strong solution to $M u=f$ is a function

$$
u \in W^{1,1}\left([-1,0] \times S^{d-1}\right) \cap W_{\text {loc }}^{2, \infty}\left((-1,0) \times S^{d-1}\right)
$$

which satisfies the PDE $M u=f$ pointwise almost everywhere on $(-1,0) \times S^{d-1}$. Such a function satisfies the boundary conditions by having its derivatives of first order $\left(u_{z}\right.$ and $\left.u_{\theta}\right)$ bounded. This class of solutions has good compactness properties for weak* convergence.

Lemma 7.2. If $\sup _{z, \theta} \ell(z, \theta)<0$, then any strong solution $u$ to $M u=0$ vanishes.

Proof. We construct a supersolution of the form $\bar{u}(z, \theta)=U(z)$. By assumption a constant $0<a<1$ exists such that

$$
A_{00}(z, \theta) \leq a^{-1}, \quad \ell(z, \theta) \leq-a
$$

for all $(z, \theta)$, and such that the following holds for certain $-1<z_{1}<z_{2}<0$ :

$$
k_{0}(z, \theta)\left\{\begin{array}{l}
\geq a \text { for }-1 \leq z \leq z_{1} \\
\leq-a \text { for } z_{2} \leq z \leq 0
\end{array}\right.
$$


We choose $U(z)=1$ for $z_{1} \leq z \leq z_{2}$ and we will construct $U$ so that $U$ is convex $\left(U^{\prime \prime} \geq 0\right)$, strictly decreasing on $\left(-1, z_{1}\right]$, and strictly increasing on $\left[z_{2}, 0\right)$. For such $U$ one has

$$
\begin{aligned}
M U & =(1+z) z^{2} A_{00}(z, \theta) U^{\prime \prime}(z)+k_{0}(z, \theta d) U^{\prime}(z)+\ell(z, \theta) U(z) \\
& \leq \begin{cases}a\left\{a^{-2}(1+z) z^{2} U^{\prime \prime}(z)+U^{\prime}(z)-U(z)\right\} & \text { for }-1<z<z_{1}, \\
-a \text { for } z_{1}<z<z_{2} & \\
a\left\{a^{-2}(1+z) z^{2} U^{\prime \prime}(z)-U^{\prime}(z)-U(z)\right\} & \text { for } z_{2}<z<0 .\end{cases}
\end{aligned}
$$

For $z \in\left(-1, z_{1}\right)$ we define $U$ to be the solution of

$$
a^{-2}(1+z) z^{2} U^{\prime \prime}(z)+U^{\prime}(z)-\frac{1}{2} U(z)=0, \quad U\left(z_{1}\right)=1, U^{\prime}\left(z_{1}\right)=0,
$$

and for $z \in\left(z_{2}, 0\right)$ we let $U$ be the solution of

$$
a^{-2}(1+z) z^{2} U^{\prime \prime}(z)-U^{\prime}(z)-\frac{1}{2} U(z)=0, \quad U\left(z_{2}\right)=1, U^{\prime}\left(z_{2}\right)=0 .
$$

One can easily verify that $U$ thus defined is indeed decreasing on $\left(-1, z_{1}\right)$ and increasing on $\left(z_{2}, 0\right)$, and by the differential equations that $U$ is indeed convex. Moreover one finds that

$$
\lim _{z \searrow-1} U^{\prime}(z)=-\infty \quad \text { and } \quad \lim _{z \nearrow 0} U^{\prime}(z)=\infty
$$

In fact, $z=-1$ is a regular singular point with exponents $r_{1}=0, r_{2}=1-a^{2}$, so there is one analytic solution and one solution whose derivative at $z=-1$ grows like $(1+z)^{-a^{2}}$. At $z=0$ we have an irregular singular point near which $U>0$ so that $a^{-2}(1+z) z^{2} U^{\prime \prime}(z)>U^{\prime}(z)$. Integration shows that $U^{\prime}(z)$ grows like $e^{-c / z}$ for some $c>0$, as $z \nearrow 0$.

The function $U$ as constructed here satisfies $M(U)<0$ pointwise for $-1<z<0$.

Now let $u \in W^{1,1}\left([-1,0] \times S^{d-1}\right) \cap W_{\text {loc }}^{2, \infty}\left((-1,0) \times S^{d-1}\right)$ be a strong solution to $M u=0$. Then for any $\varepsilon>0$ one has $M(u-\varepsilon U)=-\varepsilon M(U)>0$. This equation is uniformly elliptic on the domain $\Omega_{\delta}=[-1+\delta,-\delta] \times S^{d-1}$. Since $u_{z}$ is bounded, we have $(u-\varepsilon U)_{z}>0$ for $z$ close to $-1+\delta$ and $(u-\varepsilon U)_{z}>0$ for $z$ close to $-\delta$. Therefore $u-\varepsilon U$ does not attain its maximum on the boundary of the region $\Omega_{\delta}$ and the maximum principle for strong solutions [15, Theorem 9.1] implies $u-\varepsilon U \leq 0$ on $\Omega_{\delta}$. This holds for any positive $\varepsilon$ and $\delta$ so we see that $u \leq 0$ on $(-1,0) \times S^{d-1}$. Repeating the whole argument for $-u$ we see that $u=0$.

Lemma 7.3. If $\sup _{z, \theta} \ell(z, \theta)<0$, then a constant $C<\infty$ exists such that $\|u\|_{Y} \leq$ $C\|M u\|_{X}$ holds for all $u \in Y$.

Proof. We argue by contradiction. Let $u_{k} \in Y$ be a sequence of functions with $\left\|u_{k}\right\|_{Y}=1$ and $\left\|M u_{k}\right\|_{X} \rightarrow 0$. We may then extract a subsequence (which we again denote by $\left.u_{k}\right)$ which converges in the weak* topologies of $W^{2, \infty}\left((-1,0) \times S^{d-1}\right)$ and $W^{1,1}\left([-1,0] \times S^{d-1}\right)$. The limit $u_{*}$ is a strong solution of $M u_{*}=0$ and hence must vanish.

On the other hand we have $1=\left\|u_{k}\right\|_{Y} \leq C\left(\left\|u_{k}\right\|_{X}+\left\|M u_{k}\right\|_{X}\right)$ so that $\left\|u_{k}\right\|_{X} \geq$ $C^{-1}-o(1)$. The embedding of $W^{1,1}\left([-1,0] \times S^{d-1}\right)$ in $X$ is compact so weak* convergence in $W^{1,1}\left([-1,0] \times S^{d-1}\right)$ turns into norm convergence in $X$. We get $\left\|u_{*}\right\|_{X} \geq C^{-1}$, which contradicts $u_{*}=0$. 
The method of continuity [15. Theorem 5.2] now tells us that we only have to show that one of the operators $M$ with $\ell(z, \theta)<0$ is surjective in order to conclude that they all are. In fact, the inequality $\|u\|_{Y} \leq C\|M u\|_{X}$ which we have just proved implies that the range of $M$ is closed, so we merely have to prove solvability of $M u=f$ for a set of $f^{\prime}$ 's which is dense in $X$. Our choice is

$$
\bar{M}=(1+z)\left\{z^{2} \partial_{z}^{2}+\Delta_{\theta}\right\}-(1+2 z) \partial_{z}-1 .
$$

This operator is invariant under rotations of $S^{d-1}$ and hence commutes with the heat semigroup $e^{\varepsilon \Delta_{\theta}}$.

Lemma 7.4. The equation $\bar{M} u=f$ has a solution $u \in Y$ for any $f \in X$ which is of the form $f=e^{\varepsilon \Delta_{\theta}} g$, with $\left.g \in C^{\infty}\left([-1,0] \times S^{d-1}\right]\right)$.

Proof. We first construct a solution $v$ to $\bar{M} v=g$. The constant functions $\pm\|g\|_{\infty}$ are sub- and super solutions, and one can solve the Dirichlet problem $\bar{M} v=g$ on any compact $\bar{\Omega} \subset(-1,0) \times S^{d-1}$ with smooth boundary since the equation is non-degenerate on such a region. Hence Perron's method works and provides us with a solution $v \in C^{\infty}\left((-1,0) \times S^{d-1}\right)$ which is also bounded by $|v| \leq\|g\|_{\infty}$.

Now we consider $u=e^{\varepsilon \Delta_{\theta}} v$. Boundedness of $v$ and the smoothing effect of the heat semigroup causes this function to have bounded derivatives of all orders with respect to the angle variables. It also satisfies $\bar{M} u=e^{\varepsilon \Delta_{\theta}} g$. We rewrite the PDE as an ODE in which the angle variable $\theta$ occurs as a parameter:

$$
(1+z) z^{2} u_{z z}-(1+2 z) u_{z}=u+e^{\varepsilon \Delta_{\theta}} g \stackrel{\text { def }}{=} h(z, \theta) .
$$

Integration of this ODE gives

$$
u_{z}(z, \theta)=u_{z}\left(-\frac{1}{2}, \theta\right) e^{-2+1 / z} \frac{1+z}{z}+\int_{-1 / 2}^{z} \frac{e^{1 / z-1 / z^{\prime}}}{z z^{\prime}} \frac{1+z}{\left(1+z^{\prime}\right)^{2}} h\left(z^{\prime}, \theta\right) d z^{\prime}
$$

A careful study of the singularities at $z=0$ and $z=-1$ in the integral shows that

$$
\sup _{-1<z<0}\left|\int_{-1 / 2}^{z} \frac{e^{1 / z-1 / z^{\prime}}}{z z^{\prime}} \frac{1+z}{\left(1+z^{\prime}\right)^{2}} d z^{\prime}\right|=A<\infty
$$

and hence (7.2) implies for each $\theta \in S^{d-1}$

$$
\sup _{-1<z<0}\left|u_{z}(z, \theta)\right| \leq C\left|u_{z}\left(-\frac{1}{2}, \theta\right)\right|+A \sup _{-1<z<0}|h(z, \theta)| .
$$

One can differentiate (7.2) with respect to the angle $\theta$ as often as one likes, and so we also get $L^{\infty}$ bounds for $\partial_{\theta}^{k} u_{z}$ for all $k \geq 1$,

$$
\sup _{-1<z<0}\left|\partial_{z} \partial_{\theta}^{k} u(z, \theta)\right| \leq C\left|\partial_{\theta}^{k} u_{z}\left(-\frac{1}{2}, \theta\right)\right|+A \sup _{-1<z<0}\left|\partial_{\theta}^{k} h(z, \theta)\right| .
$$

These bounds imply that the angle derivatives $\partial_{\theta}^{k} u(z, \theta)$ themselves have limits as $z \nearrow 0$ or $z \searrow-1$. The term $h=u+e^{\varepsilon \Delta_{\theta}} g$ and its angle derivatives then also have limits at $z=0$ and $z=-1$. Using the integral in (7.2) again we see that $u_{z}$ has limits at $z=0,-1$, as do its derivatives $\partial_{\theta}^{k} u_{z}$.

So far we can conclude that $u, u_{z}, u_{\theta}, u_{\theta \theta}$, and $u_{z \theta}$ belong to

$$
X=h^{0, \alpha}\left([-1,0] \times S^{d-1}\right) .
$$

The PDE then implies that the remaining term $(1+z) z^{2} u_{z z}$ also belongs to $X$ so that $u$ belongs to $Y$, so that we can indeed solve $\bar{M} u=e^{\varepsilon \Delta_{\theta}} g$ for any $g \in C^{\infty}$ with $u \in X$. 
Since any $f \in X$ can be approximated by functions of the form $e^{\varepsilon \Delta_{\theta}} g$ with $g \in C^{\infty}$, we have proved that the range of $\bar{M}$ is dense, and thus that all $M$ with $\ell<0$ are bijections from $Y$ to $X$. This also completes the proof of the Fredholm property for general $M$ with sign changing $\ell$.

\section{Linearization at the AG SOlution}

Now that we have established that self-similar hole filling solutions of the PME are solutions of a non-linear analytic Fredholm equation we can apply well-known methods from bifurcation theory [12], 16]. We first recall the Lyapunov-Schmidt construction which reduces the equation $\mathfrak{f}(N, a, m)=0$ locally to a finite set of analytic equations. Then we apply this construction to the radially symmetric solutions found by Aronson and Graveleau, and we show that a sequence of symmetry breaking bifurcations must occur as the parameter $m$ decreases from $m=\infty$ to $m=1$.

8.1. Computing the derivatives of $\mathfrak{f}$ and $\mathfrak{g}$. In this section we will use the following notation:

$$
\mathfrak{g}(P, a, m)=\text { right-hand side of }(3.9),
$$

so that we have

$$
\mathfrak{f}(N, a, m)=L^{\prime}(\eta) \mathfrak{g}(c L(\eta)+N, a, m) .
$$

This leads to the following relations of the derivatives of $\mathfrak{f}$ and $\mathfrak{g}$ :

$$
\begin{aligned}
& \mathfrak{f}_{N}(N, a, m) \cdot \delta N=L^{\prime}(\eta) \mathfrak{g}_{P}(P, a, m) \cdot \delta P \\
& \mathfrak{f}_{a}(N, a, m)=L^{\prime}(\eta) \mathfrak{g}_{a}(P, a, m)-L^{\prime}(\eta) \frac{\mathfrak{g}_{P}(P, a, m) \cdot L(\eta)}{(2 a-1)^{2}},
\end{aligned}
$$

and

$$
\mathfrak{f}_{m}(N, a, m)=L^{\prime}(\eta) \mathfrak{g}_{m}(P, a, m) .
$$

Recall that $P, c$, and $N$ are related by (4.1), i.e.

$$
P(\eta, \theta)=c L(\eta)+N(\eta, \theta), \quad \text { with } c=\frac{a}{2 a-1} .
$$

The operator $\mathfrak{f}_{N}$, being the Fréchèt derivative of a smooth mapping, is a priori only defined as a bounded linear operator from $Y$ to $X$, but since this operator is represented by a second order elliptic differential operator it can be extended to a linear operator defined on, say, $C_{\text {loc }}^{2}\left(\mathbb{R}_{+} \times S^{d-1}\right)$. We will abuse notation and use $\mathfrak{f}_{N}$ for both the Fréchèt derivative and its extension. Since we have not specified the domain of $\mathfrak{g}$ we cannot interpret $\mathfrak{g}_{P}$ as a Fréchèt derivative, and accordingly we always let $\mathfrak{g}_{P}$ stand for the partial differential operator obtained by formally differentiating $\mathfrak{g}(P, a, m)$ with respect to $P$.

We now assume that $\bar{P}=\bar{P}(\eta)$ is a radially symmetric solution of $\mathfrak{g}(P, a, m)=$ 0 with $\bar{P}(0)=0$. The existence and uniqueness of one such solution for every $m \in(1, \infty)$ was established by Aronson and Graveleau [17, 6].

For radially symmetric $\bar{P}$ the expressions for the derivatives $\mathfrak{g}_{P}, \mathfrak{g}_{a}$, etc. simplify. After a computation one ends up with these formulae:

$$
\left.\mathfrak{g}_{P}(\bar{P}, a, m) \cdot \phi \stackrel{\text { def }}{=} \frac{\partial \mathfrak{g}(\bar{P}+\varepsilon \phi, a, m)}{\partial \varepsilon}\right|_{\varepsilon=0}
$$


So

$$
\mathfrak{g}_{P}(\bar{P}, a, m)=A(\eta)\left(\frac{\partial}{\partial \eta}\right)^{2}+B(\eta) \frac{\partial}{\partial \eta}+\eta \Delta_{\theta}+C(\eta)
$$

where

$$
\left\{\begin{array}{l}
A(\eta)=\eta / \bar{P}^{\prime}(\eta)^{2} \\
B(\eta)=-\frac{2 \eta \bar{P}^{\prime \prime}(\eta)}{\bar{P}^{\prime}(\eta)^{3}}+\frac{1}{(m-1) \bar{P}^{\prime}(\eta)^{2}}-\frac{2 a-1}{m-1} \eta e^{2 \bar{P}(\eta)} \\
C(\eta)=-\frac{2}{m-1}\left\{(2 a-1) \eta \bar{P}^{\prime}(\eta)-a\right\} e^{2 \bar{P}(\eta)}
\end{array}\right.
$$

For $\eta \rightarrow \infty$ the coefficients $A, B, C$ satisfy

$$
\left\{\begin{array}{l}
A(\eta)=A_{0} \eta^{3}+A_{1} \eta^{3-\frac{1}{2 a-1}}+A_{2} \eta^{3-\frac{2}{2 a-1}}+\cdots \\
B(\eta)=-B_{0} \eta^{2+\frac{1}{2 a-1}}+A_{1} \eta^{2}+A_{2} \eta^{2-\frac{1}{2 a-1}}+\cdots \\
C(\eta)=C_{0} \eta+o(\eta)
\end{array}\right.
$$

where $A_{0}$ and $B_{0}$ are positive constants.

At $\eta=0$ one has

$$
\left\{\begin{array}{l}
A(\eta)=a^{2} \eta+\mathcal{O}\left(\eta^{2}\right) \\
B(\eta)=\frac{a^{2}}{m-1}+\mathcal{O}(\eta) \\
C(\eta)=\frac{2 a}{m-1}+\mathcal{O}(\eta)
\end{array}\right.
$$

Differentiation with respect to $a$ gives

$$
\mathfrak{g}_{a}(\bar{P}, a, m)=-\frac{e^{2 \bar{P}}}{m-1}\left(2 \eta \bar{P}^{\prime}(\eta)-1\right)
$$

and the derivative with respect to $m$ is

$$
\mathfrak{g}_{m}(\bar{P}, a, m)=\frac{1}{(m-1)^{2}}\left\{\frac{1}{\bar{P}_{\eta}}+e^{2 \bar{P}}\left[(2 a-1) \eta \bar{P}_{\eta}-a\right]\right\} .
$$

8.2. Scaling. The set of self-similar solutions is invariant under the scaling (2.2) so that self-similar solutions come in one-parameter families given by (2.2). In our present context this is expressed by the following identity:

$$
\mathfrak{g}\left(\mathfrak{S}^{\lambda} P-\frac{1}{2} \ln \lambda, a, m\right)=\frac{1}{\lambda} \mathfrak{S}^{\lambda}\{\mathfrak{g}(P, a, m)\},
$$

where $\mathfrak{S}^{\lambda} u(\eta, \theta)=u(\lambda \eta, \theta)$. In particular, any solution $P$ of $\mathfrak{g}(P, a, m)=0$ gives rise to a family of solutions

$$
P^{\lambda}(\eta, \theta)=P(\lambda \eta, \theta)-\frac{1}{2} \ln \lambda \quad(0<\lambda<\infty) .
$$

Lemma 8.1. Let $(N, a, m) \in \mathcal{O}$ be a solution of $\mathfrak{f}(N, a, m)=0$. Then $2 \eta P_{\eta}-1 \in$ $\operatorname{ker} \mathfrak{f}_{N}$. 
Proof. For all $\lambda>0$ the function $P^{\lambda}(\eta, \theta)=P(\lambda \eta, \theta)-\frac{1}{2} \ln \lambda$ also satisfies $\mathfrak{g}\left(P^{\lambda}, a, m\right)=0$. Differentiate with respect to $\lambda$ at $\lambda=1$ to get $\mathfrak{g}_{P}(P, a, m)$. $\left(2 \eta P_{\eta}-1\right)=0$, so that $2 \eta P_{\eta}-1$ satisfies the differential equation defining ker $\mathfrak{f}_{N}$. It remains to verify that $2 \eta P_{\eta}-1$ also belongs to $Y$, i.e. that it is sufficiently regular at $\eta=0$ and $\eta=\infty$. At $\eta=0$ we know that $P$ is smooth, so that $2 \eta P_{\eta}-1$ is also smooth. At $\eta=\infty$ we compute the asymptotic expansion

$$
\begin{aligned}
2 \eta P^{\prime}(\eta, \theta)-1 & =\frac{1}{2 a-1}+c_{1}(\theta) \eta^{\frac{-1}{2 a-1}}+c_{2}(\theta) \eta^{\frac{-2}{2 a-1}}+\cdots \\
& =\frac{1}{2 a-1}-c_{1}(\theta) z+c_{2}(\theta) z^{2}+\cdots,
\end{aligned}
$$

which shows that $2 \eta P_{\eta}(\eta, \theta)-1$ does indeed belong to $Y$.

We will eliminate this trivial multiplicity of solutions by imposing a further normalizing condition on the solution $P$, namely

$$
\int_{S^{d-1}} P(0, \theta) d \theta=0
$$

and we define

$$
Y_{0}=\{N \in Y: N \text { satisfies }(8.9)\} .
$$

We will look for solutions to $\mathfrak{f}(N, a, m)=0$ in the space

$$
\mathcal{O}_{0}=\left\{(N, a, m) \in \mathcal{O}: N \in Y_{0}\right\} .
$$

Since $Y_{0}$ has codimension one in $Y$, the linear map $\mathfrak{f}_{N} \mid Y_{0}: Y_{0} \rightarrow X$ has Fredholm index one less than $\mathfrak{f}_{N}: Y \rightarrow X$, i.e. $\mathfrak{f}_{N} \mid Y_{0}$ has index -1 . The non-linear map $\mathfrak{f}: \mathcal{O}_{0} \rightarrow X$ has index $2-1=1$; generically one would expect its zeroset to consist of smooth curves without singular points. However our map is not generic because of the rotational symmetry which underlies the problem.

8.3. Symmetries and separation of variables. The group $O(d, \mathbb{R})$ of $d \times d$ orthogonal matrices acts on $\mathbb{R}^{d}$ and leaves $S^{d-1}$ invariant. The equation for selfsimilar solutions is also invariant under this action. Formally, if we define

$$
\mathfrak{R}_{T} u(\eta, \theta)=u(\eta, T \theta) \quad \text { for } T \in O(d, \mathbb{R}),
$$

then

$$
\mathfrak{f}\left(\mathfrak{R}_{T} N, a, m\right)=\mathfrak{R}_{T} \mathfrak{f}(N, a, m) .
$$

If $\bar{N}$ is a radial solution to $\mathfrak{f}(N, a, m)=0$, i.e. a solution which only depends on $\eta$, then $\mathfrak{R}_{T} \bar{N}=\bar{N}$ for all $T \in O(d, \mathbb{R})$. Differentiating (8.10) with respect to $N$ we find that

$$
\mathfrak{f}_{N}(\bar{N}, a, m) \cdot \mathfrak{R}_{T}=\mathfrak{R}_{T} \cdot \mathfrak{f}_{N}(\bar{N}, a, m) .
$$

The linear operator $\mathfrak{f}_{N}$ at a radial solution therefore commutes with the group action, and also with the spherical Laplacian $\Delta_{\theta}$ as one could also simply see from (8.4).

Recall that the spherical Laplacian has eigenvalues $-l(l+d-2)$ with $l \in \mathbb{N}_{0}$ and that its eigenfunctions are called the "spherical harmonics". For each $l \in \mathbb{N}_{0}$ one defines $y: S^{d-1} \rightarrow \mathbb{R}$ to be a spherical harmonic of degree $l$ if the function

$$
u: x \in \mathbb{R}^{d} \mapsto|x|^{l} \mathrm{y}\left(\frac{x}{|x|}\right)
$$


is a polynomial of degree $l$ which satisfies $\Delta_{\mathbb{R}^{d}} u(x)=0$. The space $H_{l}\left(S^{d-1}\right)$ of spherical harmonics of degree $l$ has dimension

$$
n=n_{d, l}=\left(\begin{array}{c}
d+l-2 \\
l
\end{array}\right)+\left(\begin{array}{c}
d+l-3 \\
l-1
\end{array}\right)
$$

and we will let $\left\{y_{l}^{(1)}(\theta), \ldots, y_{l}^{(n)}(\theta)\right\}$ be a basis for this space.

The spaces $H_{l}\left(S^{d-1}\right)$ are mutually orthogonal for the $L^{2}\left(S^{d-1}\right)$ inner product. We choose our basis $\left\{y_{l}^{(i)}(\theta)\right\}$ to be orthogonal for the same inner product.

In the two-dimensional situation we have $d=2$, so that $n=2$ for all $l \geq 1$. In this case we can represent the unit vector $\theta \in \mathbb{R}^{2}$ as a complex number $\theta=e^{i \omega}$ and the spherical harmonics are just the usual trigonometric functions

$$
y_{l}^{(1)}\left(e^{i \omega}\right)=\cos l \omega, \quad y_{l}^{(2)}\left(e^{i \omega}\right)=\sin l \omega .
$$

We decompose the space $X$ into eigenspaces of the spherical Laplacian. Let $X^{l} \subset X$ be the subspace of functions of the form

$$
\phi(\eta, \theta)=\Phi_{1}(\eta) y_{l}^{(1)}(\theta)+\cdots+\Phi_{n}(\eta) y_{l}^{(n)}(\theta)
$$

with $\Phi_{j}(\eta) \in C\left([0, \infty]\right.$ ), and where $n=n_{d, l}$ (we will stick to this abbreviation from here on). We then have, in a weak sense,

$$
X=X^{0} \oplus X^{1} \oplus X^{2} \oplus \cdots .
$$

In other words, the spaces $X^{j}$ are mutually disjoint $\left(X^{j} \cap X^{i}=(0)\right.$ if $\left.i \neq j\right)$ and the span of the $X^{j}$ is dense in $X$. Any $\phi \in X^{l}$ satisfies

$$
-\Delta_{\theta} \phi=l(l+d-2) \phi .
$$

Functions in $X^{0}$ do not depend on $\theta$ and we will use the alternative notation $X^{\mathrm{rad}}=X^{0}$.

We will also consider the derived spaces

$$
\begin{aligned}
Y^{l} & =Y \cap X^{l}, \quad Y_{0}^{l}=Y_{0} \cap X^{l}, \\
\mathcal{O}^{l} & =\left\{(N, a, m) \in \mathcal{O}: N \in Y^{l}\right\}, \quad \text { etc. }
\end{aligned}
$$

Since the operator $\mathfrak{f}_{N}(\bar{N}, a, m)$ commutes with the spherical Laplacian, it maps $Y^{l}$ into $X^{l}$.

8.4. Formal self-adjointness of $\mathfrak{f}_{N}$. The differential operator $\mathfrak{f}_{N}$ is formally a self-adjoint operator in a suitably weighted $L^{2}$ space. If we define

$$
\sigma(\eta)=\frac{1}{L^{\prime}(\eta) A(\eta)} \exp \int_{1}^{\eta} \frac{B\left(\eta^{\prime}\right)}{A\left(\eta^{\prime}\right)} d \eta^{\prime}
$$

then $\sigma$ satisfies

$$
\frac{d}{d \eta}\left(L^{\prime}(\eta) A(\eta) \sigma(\eta)\right)=B(\eta) \sigma(\eta)
$$

Let

$$
Z_{\sigma}=L^{2}\left(\mathbb{R}_{+} \times S^{d-1}, \sigma(\eta) d \eta d \theta\right)
$$

be the Hilbert space with inner product

$$
\langle\phi, \psi\rangle_{\sigma}=\int_{S^{d-1}} \int_{0}^{\infty} \sigma(\eta) \phi(\eta, \theta) \psi(\eta, \theta) d \eta d \theta .
$$


For $\phi, \psi \in C_{c}^{\infty}\left(\mathbb{R}_{+} \times S^{d-1}\right)$ one integrates by parts to get

$$
\begin{aligned}
\left\langle\phi, \mathfrak{f}_{N}(\bar{N}, a, m) \psi\right\rangle_{\sigma} & =\int_{S^{d-1}} \int_{0}^{\infty} \sigma \phi L^{\prime}(\eta)\left\{A \psi_{\eta \eta}+B \psi_{\eta}+\eta \Delta_{\theta} \psi+C \psi\right\} d \eta d \theta \\
& =-\int_{S^{d-1}} \int_{0}^{\infty} \sigma L^{\prime}(\eta)\left\{A \phi_{\eta} \psi_{\eta}+\eta \nabla_{\theta} \phi \cdot \nabla_{\theta} \psi-C \phi \psi\right\} d \eta d \theta
\end{aligned}
$$

which is symmetric in $\phi$ and $\psi$. It turns out that this identity holds for a much wider class of functions $\phi, \psi$.

Lemma 8.2. If $\phi, \psi \in C_{\mathrm{loc}}^{2}\left([0, \infty) \times S^{d-1}\right)$ satisfy

$$
|\phi|+|\psi|+\left|\phi_{\eta}\right|+\left|\psi_{\eta}\right| \leq C e^{\eta^{p}}
$$

for some $0<p<\frac{1}{2 a-1}$, then $\left\langle\phi, \mathfrak{f}_{N}(\bar{N}, a, m) \psi\right\rangle_{\sigma}=\left\langle\mathfrak{f}_{N}(\bar{N}, a, m) \phi, \psi\right\rangle_{\sigma}$.

Since $\frac{1}{2 a-1}>1$, this lemma allows functions $\phi$ and $\psi$ with faster than exponential growth. The proof will show that the assumption that $\phi$ and $\psi$ are regular at $\eta=0$ could also be relaxed.

Proof. We begin by looking at the asymptotic growth of $\sigma(\eta)$ for large $\eta$. One has $B(\eta) / A(\eta)=-c_{0} \eta^{\frac{1}{2 a-1}-1}+c_{1} \eta^{-1}+\cdots$ where $c_{0}>0$, and thus

$$
A(\eta) L^{\prime}(\eta) \sigma(\eta)=\exp \int_{1}^{\eta} \frac{B}{A} d \eta^{\prime} \leq \exp \left[-c^{\prime} \eta^{1 /(2 a-1)}\right] .
$$

At $\eta=0$ one has $B / A=\frac{1}{m-1} \frac{1}{\eta}+\cdots$ so that $A L^{\prime} \sigma=\eta^{1 /(m-1)}(1+o(1))$.

If we repeat the integration by parts, keeping track of the boundary terms this time we get

$$
\left\langle\phi, \mathfrak{f}_{N} \cdot \psi\right\rangle_{\sigma}-\left\langle\mathfrak{f}_{N} \cdot \phi, \psi\right\rangle_{\sigma}=\lim _{\delta \searrow 0} \lim _{R \nearrow \infty} \int_{S^{d-1}}\left[A L^{\prime} \sigma\left(\phi \psi_{\eta}-\phi_{\eta} \psi\right)\right]_{\eta=\delta}^{R} d \theta=0
$$

since $\eta^{p}=o\left(\eta^{1 /(2 a-1)}\right)$.

8.5. Consequences of formal self-adjointness. If $Y$ and $X$ were Hilbert spaces, and if $\mathfrak{f}_{N}$ were an honest self-adjoint operator, then one would have $\left(\text { range } \mathfrak{f}_{N}\right)^{\perp}=$ $\operatorname{ker} \mathfrak{f}_{N}^{*}=\operatorname{ker} \mathfrak{f}_{N}$. We now observe that even though $X$ and $Y$ are merely Banach spaces, $\operatorname{ker} \mathfrak{f}_{N}$ still complements range $\mathfrak{f}_{N}$.

Lemma 8.3. At a radial solution $\bar{N}$ of $\mathfrak{f}(N, a, m)=0$ one has

$$
\text { range } \mathfrak{f}_{N} \oplus \operatorname{ker} \mathfrak{f}_{N}=X \text {. }
$$

Proof. Since $\mathfrak{f}_{N}$ has Fredholm index zero we have codim range $\mathfrak{f}_{N}=\operatorname{dim} \operatorname{ker} \mathfrak{f}_{N}$, so we only have to show that the unique $\phi$ in the intersection of range $\mathfrak{f}_{N}$ and ker $\mathfrak{f}_{N}$ is $\phi=0$. So assume that $\phi=\mathfrak{f}_{N} \psi$ belongs to the null-space of $\mathfrak{f}_{N}$. Then we have

$$
\langle\phi, \phi\rangle_{\sigma}=\left\langle\phi, \mathfrak{f}_{N} \cdot \psi\right\rangle_{\sigma}=\left\langle\mathfrak{f}_{N} \cdot \phi, \psi\right\rangle_{\sigma}=0,
$$

which indeed implies that $\phi=0$.

Lemma 8.4. If $\bar{N}$ is a radial solution of $\mathfrak{f}(N, a, m)=0$, then

$$
\mathfrak{f}_{a}(\bar{N}, a, m) \notin \operatorname{range} \mathfrak{f}_{N}(\bar{N}, a, m) .
$$


Proof. One has $\psi=1-2 \eta \bar{P}_{\eta} \in \operatorname{ker} \mathfrak{f}_{N}(\bar{N}, a, m)$, and one has the following expression for $\mathfrak{f}_{a}$ :

$$
\begin{aligned}
\mathfrak{f}_{a}(\bar{N}, a, m) & =L^{\prime}(\eta) \mathfrak{g}_{a}(P, a, m)-\frac{L^{\prime}(\eta)}{(2 a-1)^{2}} \mathfrak{g}_{P}(P, a, m) \cdot L(\eta) \\
& =\frac{L^{\prime}(\eta) e^{2 \bar{P}}}{m-1} \psi-\frac{1}{(2 a-1)^{2}} \mathfrak{f}_{N}(P, a, m) \cdot L(\eta)
\end{aligned}
$$

by (8.1), (8.2), and 8.8). Neither term belongs to $Y$, but then neither term grows faster than some power of $\eta$ as $\eta \nearrow \infty$, so we can use the formal self-adjointness of $\mathfrak{f}_{N}$ to compute

$$
\begin{aligned}
\left\langle\psi, \mathfrak{f}_{a}\right\rangle_{\sigma} & =\left\langle\psi, \frac{L^{\prime}(\eta) e^{2 \bar{P}}}{m-1} \psi-\frac{1}{(2 a-1)^{2}} \mathfrak{f}_{N} \cdot L(\eta)\right\rangle_{\sigma} \\
& =\frac{1}{m-1}\left\langle\psi, L^{\prime}(\eta) e^{2 \bar{P}} \psi\right\rangle_{\sigma}-\frac{\left\langle\psi, \mathfrak{f}_{N} \cdot L(\eta)\right\rangle_{\sigma}}{(2 a-1)^{2}} \\
& =\frac{1}{m-1}\left\langle\psi, L^{\prime}(\eta) e^{2 \bar{P}} \psi\right\rangle_{\sigma}-\frac{\left\langle\mathfrak{f}_{N} \cdot \psi, L(\eta)\right\rangle_{\sigma}}{(2 a-1)^{2}} \\
& =\frac{1}{m-1}\left\langle\psi, L^{\prime}(\eta) e^{2 \bar{P}} \psi\right\rangle_{\sigma} \\
& >0 .
\end{aligned}
$$

On the other hand, if $\mathfrak{f}_{a}$ belonged to range $\mathfrak{f}_{N}$, then one would have $\left\langle\psi, \mathfrak{f}_{a}\right\rangle_{\sigma}=0$ so this is clearly not the case.

Lemma 8.5. Let $\bar{N}$ be a radial solution, and let $\left\{\phi_{1}, \ldots, \phi_{n}\right\}$ be a basis of $\operatorname{ker} \mathfrak{f}_{N}(\bar{N}, a, m) \mid Y_{0}$. Then the space spanned by $\left\{1-2 \eta \bar{P}^{\prime}(\eta), \phi_{1}, \ldots, \phi_{n}\right\}$ is a complement for range $\mathfrak{f}_{N}(\bar{N}, a, m) \mid Y_{0}$.

Proof. The average of $1-2 \eta P^{\prime}(\eta)$ over $\theta \in S^{d-1}$ at $\eta=0$ is 1 , so $1-2 \eta P^{\prime}(\eta) \in Y \backslash Y_{0}$ and we have $Y=Y_{0} \oplus \operatorname{Span}\left\{1-2 \eta \bar{P}^{\prime}(\eta)\right\}$. Since $1-2 \eta P^{\prime}(\eta)$ is in the null-space of $\mathfrak{f}_{N}(\bar{N}, a, m)$, this implies that range $\mathfrak{f}_{N}(\bar{N}, a, m) \mid Y_{0}=\operatorname{range}_{N}(\bar{N}, a, m)$, while we also have $\operatorname{ker} \mathfrak{f}_{N}(\bar{N}, a, m)=\operatorname{ker} \mathfrak{f}_{N}(\bar{N}, a, m) \mid Y_{0} \oplus \operatorname{Span}\left\{1-2 \eta \bar{P}^{\prime}(\eta)\right\}$. By (8.14) we get

$$
X=\operatorname{range}_{N}(\bar{N}, a, m)\left|Y_{0} \oplus \operatorname{ker} \mathfrak{f}_{N}(\bar{N}, a, m)\right| Y_{0} \oplus \operatorname{Span}\left\{1-2 \eta \bar{P}^{\prime}(\eta)\right\}
$$

as claimed.

8.6. Spectral theory for $\mathfrak{f}_{N}$. We may regard the operator $\mathfrak{f}_{N}(\bar{N}, a, m)$ as an unbounded operator in the Banach space $X$ with domain $Y$.

Since the embedding $I: Y \hookrightarrow X$ is compact, $\mathfrak{f}_{N}-\lambda I$ is Fredholm with index 0 for any $\lambda \in \mathbb{C}$. The formal self-adjointness of $\mathfrak{f}_{N}$ implies that $\mathfrak{f}_{N}-\lambda I$ is injective if $\lambda$ is not real. Hence the spectrum of $\mathfrak{f}_{N}$ consists of isolated real eigenvalues of finite multiplicity.

Lemma 8.6. $\mathfrak{f}_{N}$ is bounded from above.

Proof. For any $\phi \in Y$ one has

$$
\left\langle\phi, \mathfrak{f}_{N} \cdot \phi\right\rangle_{\sigma}=\int_{S^{d-1}} \int_{0}^{\infty} \sigma(\eta) L^{\prime}(\eta)\left\{-A \phi_{\eta}^{2}-\eta\left|\nabla_{\theta} \phi\right|^{2}+C \phi^{2}\right\} d \eta d \theta \leq k\langle\phi, \phi\rangle_{\sigma}
$$

with $k=\sup _{0<\eta<\infty} L^{\prime}(\eta) \sigma(\eta)<\infty$. 
It follows that the spectrum of $\mathfrak{f}_{N}$ consists of a sequence of real eigenvalues of finite multiplicity which converge to $-\infty$.

The preceding remarks apply equally to the restricted operator $\mathfrak{f}_{N}(\bar{N}, a, m)$ : $Y^{l} \rightarrow X^{l}$ and show that this operator is Fredholm with index zero (since it is a compact perturbation of $\mathfrak{f}_{N}-\lambda I: Y^{l} \rightarrow X^{l}$ which is invertible if $\lambda$ is not real), that $\mathfrak{f}_{N} \mid Y^{l}$ is formally self-adjoint, bounded from above, and hence has a countable sequence of eigenvalues

$$
\lambda_{l, 0}>\lambda_{l, 1}>\lambda_{l, 2}>\cdots
$$

which accumulate at $-\infty$. The operator $\mathfrak{f}_{N} \mid Y^{l}$ is given by a scalar ordinary differential operator so that its eigenfunctions corresponding to $\lambda_{l, j}$ have the form $\phi(\eta, \theta)=\Phi(\eta) y_{l}(\theta)$, where $\Phi(\eta)$ is a solution of

$$
A(\eta) \Phi^{\prime \prime}(\eta)+B(\eta) \Phi^{\prime}(\eta)+\{C(\eta)-l(l+d-2) \eta\} \Phi(\eta)=\frac{\lambda}{L^{\prime}(\eta)} \Phi(\eta)
$$

with $A, B, C$ as in (8.5).

8.7. Sturm-Liouville analysis of the eigenfunctions. From the asymptotics at $\eta=0$ we see that the ODE (8.15) has a regular singular point at $\eta=0$, where the principal part of the equation is

$$
a^{2} \eta \Phi^{\prime \prime}+\frac{a^{2}}{m-1} \Phi^{\prime}=0,
$$

so that the characteristic exponents are $r_{1}=0$ and $r_{2}=\frac{m-2}{m-1}$. Thus there is only one solution (up to multiples) which is analytic at $\eta=0$, all other solutions have their derivative unbounded as $\eta \searrow 0$. Let $\Phi_{0}(\eta)$ denote the regular solution with $\Phi_{0}(0)=1$.

The asymptotics at $\eta=\infty$ show that 8.15 has an irregular singular point at $\eta=\infty$. Replacing the coefficients by their asymptotic forms the equation becomes

$$
A_{0} \eta^{3} \Phi^{\prime \prime}-B_{0} \eta^{2+\frac{1}{2 a-1}} \Phi^{\prime}+\left\{C_{0}-\lambda-l(l+d-2)\right\} \eta \Phi=0
$$

and we see that a solution $\Phi$ has the following options at $\eta=\infty$. Most solutions will try to balance the first two terms, i.e. they will satisfy $\Phi^{\prime \prime} / \Phi^{\prime}=B_{0} / A_{0} \eta^{\frac{1}{2 a-1}-1}$ which implies that they grow superexponentially,

$$
\Phi(\eta)=e^{(c+0(1)) \eta^{1 /(2 a-1)}} .
$$

There is however one solution (up to multiples again) which tries to balance the second and third term in the ODE, and thus satisfies

$$
\Phi^{\prime} / \Phi=\frac{C_{0}-\lambda-l(l+d-2)}{B_{0}} \eta^{-1-\frac{1}{2 a-1}}
$$

which can be integrated and gives

$$
\Phi(\eta)=\Phi(\infty)+o(1)
$$

for some $\Phi(\infty) \neq 0$. Let $\Phi_{\infty}(\eta)$ denote the solution of (8.15) which at $\eta=\infty$ takes the value $\Phi=1$.

For most values of $\lambda$ the two solutions $\Phi_{0}, \Phi_{\infty}$ will be independent; the eigenvalues are exactly those for which $\Phi_{0}=\kappa \Phi_{\infty}$ for some $\kappa \in \mathbb{R}$. Let $\Phi_{l, j}$ denote the eigenfunction which corresponds to the eigenvalue $\lambda_{l j}$. Sturm-Liouville theory implies that this eigenfunction has exactly $j$ zeros $\eta_{1}<\eta_{2}<\cdots<\eta_{j}$. 
Since the coefficient of the lowest order term in (8.15) is monotonically decreasing in $l$, the eigenvalues $\lambda_{l j}$ are also monotone in $l$. They are also monotone in $j$ so that we have

$$
l<l^{\prime} \text { implies } \lambda_{l j}>\lambda_{l^{\prime} j} \text { and } j<j^{\prime} \text { implies } \lambda_{l j}>\lambda_{l j^{\prime}} .
$$

8.8. The signs of the eigenvalues $\lambda_{l j}$. We had already found that $\psi(\eta, \theta)=$ $1-2 \eta P^{\prime}(\eta)$ belongs to the kernel of $\mathfrak{f}_{N}$. Since $\psi$ does not depend on $\theta$, it lies in $Y^{0}$ and so it is an eigenfunction corresponding to one of the $\lambda_{l j}$ with $l=0$.

Lemma 8.7. $\psi(\eta, \theta)=1-2 \eta P^{\prime}(\eta)$ has exactly one zero $\eta_{*} \in(0, \infty)$.

Proof. This follows by looking at $\xi$ in the AG phase plane. The proof is given in Section 9.1 .

Corollary 8.8. $\lambda_{0,1}=0$, and $\lambda_{l j}<0$ if $l=0$ and $j>1$, or if $l \geq 1$ and $j \geq 1$.

The only eigenvalues which can be positive are therefore $\lambda_{l j}$ with $j=0$. Since the operator $\mathfrak{f}_{N}$ is bounded from above, there can only be a finite number of positive eigenvalues, so we see that for any $m \in(1, \infty)$ an $l_{*}(m) \in \mathbb{N}$ exists such that $\lambda_{l 0}(m) \geq 0$ for $0 \leq l \leq l_{*}(m)$ and $\lambda_{l 0}(m)<0$ for $l>l_{*}(m)$. We arrive at the following picture:

\begin{tabular}{c||ccccc}
$\lambda_{l j}$ & $j=0$ & 1 & 2 & 3 & $\cdots$ \\
\hline$l=0$ & + & 0 & - & - & - \\
1 & + & - & - & - & - \\
2 & + & - & - & - & - \\
3 & + & - & - & - & - \\
$\vdots$ & $\vdots$ & $\vdots$ & $\vdots$ & $\vdots$ & $\vdots$ \\
$l_{*}-1$ & + & - & - & - & - \\
$l_{*}$ & $\geq 0$ & - & - & - & - \\
$l_{*}+1$ & - & - & - & - & - \\
SIGNS OF THE EIGENVALUES & $\lambda_{l j}(m)$
\end{tabular}

8.9. Absence of bifurcation within the class of radial solutions. Aronson and Graveleau proved for each $m \in(1, \infty)$ that there is exactly one solution $(N(m)$, $a(m), m) \in \mathcal{O}_{0}$ of $\mathfrak{f}(N, a, m)=0$. We now show that these solutions depend analytically on the parameter $m$. To this end we restrict the map $\mathfrak{f}$ to the space $\mathcal{O}_{0}^{\mathrm{rad}} \subset \mathcal{O}_{0}$ defined above. We have seen that $\mathfrak{f}: \mathcal{O}_{0}^{\mathrm{rad}} \rightarrow X^{\mathrm{rad}}$ is an analytic Fredholm mapping of index 0. At any zero of this map the nullspace of $\mathfrak{f}_{N}(N(m), a(m), m) \mid Y_{0}^{\mathrm{rad}}$ is one dimensional and is spanned by $\psi(\eta)=1-2 \eta P^{\prime}(\eta)$. It follows that the range of $\mathfrak{f}_{N}(N(m), a(m), m) \mid Y_{0}^{\mathrm{rad}}$ has codimension 1, so that

$$
\text { range } \mathfrak{f}_{N} \mid Y_{0}^{\mathrm{rad}} \oplus \operatorname{Span}\left\{\mathfrak{f}_{a}\right\}=X^{\mathrm{rad}} .
$$

The Implicit Function Theorem applies and tells us that the solutions $(N(m)$, $a(m), m) \in \mathcal{O}_{0}$ are indeed analytic functions of $m \in(1, \infty)$.

8.10. The eigenvalues $\lambda_{l j}(m)$ depend analytically on $m$. To see why this is so we recall that $\lambda_{l j}(m)$ is the $j^{\text {th }}$ eigenvalue of the operator $\mathfrak{f}_{N}: Y^{l} \rightarrow X^{l}$. These eigenvalues are not simple (except when $l=0$ ) because $\mathfrak{f}_{N}$ commutes with the group action $\left\{\mathfrak{R}_{T}: T \in O(d, \mathbb{R})\right\}$ on $X^{l}$. Standard perturbation theory [20] therefore guarantees that as one perturbs the operator $\mathfrak{f}_{N}$ the eigenvalue $\lambda_{l j}$ will 
split into $n$ eigenvalues $\lambda_{l j}^{(i)}(i=1, \ldots, n)$ and only expressions which are symmetric in the $\lambda_{l j}^{(i)}$ will depend analytically on the perturbation. However, the perturbations we consider also commute with the group action and this prevents the eigenvalue $\lambda_{l j}$ from splitting up, i.e. the $\lambda_{l j}^{(i)}$ are independent of $i$. This common value then coincides with the average

$$
\lambda_{l j}^{(i)}=\frac{1}{n}\left(\lambda_{l j}^{(1)}+\cdots+\lambda_{l j}^{(n)}\right)
$$

and therefore depends analytically on the perturbation. Consequently the $\lambda_{l j}(m)$ do depend analytically on $m$ after all.

8.11. Range and nullspace of $\mathfrak{f}_{N}-\lambda_{l j}(m) I$. For any given $m$ the eigenspace $\operatorname{ker}\left\{\mathfrak{f}_{N} \mid X^{l}-\lambda_{l j}(m) I\right\}$ is spanned by $n$ functions $\phi_{1}, \ldots, \phi_{n}$ of the form $\phi_{i}(m ; \eta, \theta)=$ $\Phi(m ; \eta) y_{l}^{(i)}(\theta)$, where $\left\{y_{l}^{(i)}(\theta)\right\}$ is our basis of $H_{l}\left(S^{d-1}\right)$. Since the eigenvalue $\lambda_{l j}(m)$ varies analytically with $m$, the eigenspace $\operatorname{ker}\left\{\mathfrak{f}_{N} \mid X^{l}-\lambda_{l j}(m) I\right\}$ does so too, and we can choose the basis $\phi_{i}(m ; \eta, \theta)$ so that it depends analytically on $m$.

Consider the adjoint $\mathfrak{f}_{N}^{*}: X^{l *} \rightarrow Y_{0}^{*}$, where $X^{l *}$ is the dual Banach space to $X^{l}$ and $Y_{0}^{*}$ is the Banach dual of $Y_{0}$. The $n$-dimensional space

$$
\operatorname{ker}\left\{\left(\mathfrak{f}_{N} \mid X^{l}\right)^{*}-\lambda_{l j}(m) I^{*}\right\} \subset X^{l *}
$$

varies analytically with $m$, and thus we can find a basis $\left\{\psi_{1}(m), \ldots, \psi_{n}(m)\right\}$ for $\operatorname{ker}\left\{\left(\mathfrak{f}_{N} \mid X^{l}\right)^{*}-\lambda_{l j}(m) I^{*}\right\}$, where the $\psi_{j}(m)$ are analytic $X^{l *}$ valued functions of $m$.

Lemma 8.9. We can choose the $\psi_{j}(m)$ so that

$$
\left\langle\psi_{j}(m), \phi_{k}(m)\right\rangle=\delta_{j k},
$$

where $\langle\psi, \phi\rangle$ denotes the duality pairing $X^{l *} \times X^{l} \rightarrow \mathbb{R}$.

Proof. It follows from the formal self-adjointness of $\mathfrak{f}_{N}$ that no linear combination of the $\phi_{k}$ lies in the range of $\mathfrak{f}_{N} \mid X^{l}-\lambda_{l j}(m) I$. Hence the matrix $a_{j k}(m)=$ $\left\langle\psi_{j}(m), \phi_{k}(m)\right\rangle$ is invertible, for if it were not, then some linear combination $\phi=$ $t_{1} \phi_{1}+\cdots+t_{n} \phi_{n}$ would satisfy $\left\langle\psi_{j}, \phi\right\rangle=0$, which would contradict

$$
\text { range }\left\{\mathfrak{f}_{N} \mid X^{l}-\lambda_{l j}(m) I\right\}={ }^{\perp}\left(\operatorname{ker}\left\{\left(\mathfrak{f}_{N} \mid X^{l}\right)^{*}-\lambda_{l j}(m) I^{*}\right\}\right) .
$$

Denote the inverse of $a_{i j}(m)$ by $a^{i j}(m)$. The basis $\psi_{j}^{\prime}(m)=\sum_{j^{\prime}} a^{j j^{\prime}}(m) \psi_{j}(m)$ satisfies (8.16) and varies analytically with $m$.

The functionals $\psi_{j}^{\prime}$ which we have constructed are only defined on $X^{l}$. We now show that they can be extended to all of $X$.

Lemma 8.10. The functionals $\psi_{j}(m) \in X^{*}$ obtained by extending the $\psi_{j}^{\prime}(m)$ so that they vanish on $X^{l^{\prime}}$ for all $l^{\prime} \neq l$ are analytic in $m$.

Proof. Let $\psi_{j}^{\prime} \in X^{l *}$ denote the functionals constructed above. Then we have $\psi_{j}(m)=\psi_{j}^{\prime}(m) \circ P^{l}$, where $P^{l}: X \rightarrow X^{l}$ is the projection which vanishes on all $X^{l^{\prime}}$ with $l^{\prime} \neq l$. This projection is given by the expression

$$
P^{l} \phi(\eta, \theta)=\sum_{i=1}^{n_{l, d}} y_{l}^{(i)}(\theta) \int_{S^{d-1}} y_{l}^{(i)}\left(\theta^{\prime}\right) \phi\left(\eta, \theta^{\prime}\right) d \theta^{\prime} \quad\left(0<\eta<\infty, \theta \in S^{d-1}\right),
$$


provided one normalizes the $y_{l}^{(i)}$ to satisfy $\int y_{l}^{(i)}(\theta)^{2} d \theta=1$. From this formula it is clear that the projection $P^{l}: X \rightarrow X^{l}$ is bounded linear, and hence analyticity of the $\psi_{j}(m)$ follows from analyticity of the $\psi_{j}^{\prime}(m)$.

We could have required the $\phi_{j}$ to be orthogonal with respect to the inner product $\left\langle\phi^{\prime}, \phi\right\rangle_{\sigma}$ on $Z_{\sigma}$, in which case the functionals would be given by

$$
\left\langle\psi_{j}(m), \phi\right\rangle=\left\langle\phi_{j}(m), \phi\right\rangle_{\sigma} .
$$

This explicit representation of the $\psi_{j}$, which comes from the formal self-adjointness of $\mathfrak{f}_{N}$, looks simpler than the construction given above, but it has the disadvantage that the $Z_{\sigma}$ inner product varies with $m$; the growth rate of the weight $\sigma$ even depends on the unknown exponent $a$; see (8.13). To use (8.17) we would therefore have to verify that this dependence is in some way analytic. By working with the dual spaces $X^{*}$, etc. we circumvent this obstacle.

8.12. Lyapunov-Schmidt reduction. If for some $m_{*} \in(1, \infty)$ one has $\lambda_{l j}\left(m_{*}\right)$ $\neq 0$ except for $\lambda_{1,0}\left(m_{*}\right)=0$, then the arguments in Section 8.9 apply verbatim, and we conclude that no bifurcation occurs at $m=m_{*}$, i.e. near $m=m_{*}$ the only solutions which are close to the (radial) AG solutions are those solutions themselves. We now assume that we are at a possible bifurcation point, namely will suppose that

$$
\lambda_{l, 0}\left(m_{*}\right)=0
$$

for some $l \geq 2$ and $m_{*} \in(1, \infty)$.

To study the solutions of $\mathfrak{f}(N, a, m)=0$ in the vicinity of $\left(N_{*}, a_{*}, m_{*}\right)$ we consider the extended system of equations

$$
\left\{\begin{array}{c}
\mathfrak{f}\left(N(m)+N^{\prime}+\sum s_{j} \phi_{j}, a(m)+a^{\prime}, m\right)+\sum r_{k} \phi_{k}=0 \\
\left\langle\psi_{1}(m), N^{\prime}\right\rangle=0 \\
\vdots \\
\left\langle\psi_{n}(m), N^{\prime}\right\rangle=0
\end{array}\right.
$$

In other words, we consider the map

$$
\mathfrak{h}: Y_{0} \times \mathbb{R}^{n} \times \mathbb{R} \times \mathbb{R} \times \mathbb{R}^{n} \rightarrow X \times \mathbb{R}^{n}
$$

which sends $\left(N^{\prime}, \mathbf{s}, a^{\prime}, m, \mathbf{r}\right)$ to the left-hand sides in (8.18) (here $\mathbf{s}=\left(s_{j}\right)_{1 \leq j \leq n}$ and $\left.\mathbf{r}=\left(r_{j}\right)_{1 \leq j \leq n}\right)$. The map $\mathfrak{h}$ is defined on an open neighborhood of $\left(N^{\prime}=0, \mathbf{s}=\mathbf{0}\right.$, $\left.a^{\prime}=0, m=m_{*}, \mathbf{r}=0\right)$.

Solutions of $\mathfrak{h}\left(N^{\prime}, \mathbf{s}, a^{\prime}, m, \mathbf{r}\right)=\mathbf{0}$ do not always correspond to solutions of $\mathfrak{f}(N, a, m)=0$, but if $\mathbf{r}$ happens to vanish, then $N=N(m)+N^{\prime}+\sum s_{j} \phi_{j}$ and $a=a(m)+a^{\prime}$ do satisfy $\mathfrak{f}(N, a, m)=0$. Conversely, if we have a solution of $\mathfrak{f}(N, a, m)=0$ which is close to our bifurcation point $\left(N_{*}, a_{*}, m_{*}\right)$, then we can split $N-N(m)=N^{\prime}+\sum s_{j} \phi_{j}$ with $\left\langle\psi_{j}, N^{\prime}\right\rangle=0$ and set $a^{\prime}=a-a(m)$ to obtain a solution $\left(N^{\prime}, \mathbf{s}, a^{\prime}, m, \mathbf{0}\right)$ of $\mathfrak{h}=0$.

We will prove in Section 8.13 that one can apply the implicit function theorem to $\mathfrak{h}$ at the bifurcation point $\left(N_{*}, a_{*}, m_{*}\right)$ and conclude that near the bifurcation point the solution set of the extended system (8.18) is given by an $n+1$ dimensional analytic manifold of the form

$$
N^{\prime}=N^{\prime}(\mathbf{s}, m), \quad a^{\prime}=a^{\prime}(\mathbf{s}, m), \quad \mathbf{r}=\mathbf{r}(\mathbf{s}, m),
$$

where $\mathbf{s}$ is close to $\mathbf{0}$ and $m$ is close to $m_{*}$. 
We call this manifold the reduced manifold. As we have just observed, all solutions to $\mathfrak{f}(N, a, m)=0$ lie on this manifold and they are singled out by the reduced equation

$$
\mathbf{r}(\mathbf{s}, m)=0,
$$

which is a set of $n$ analytic equations in $n+1$ variables.

The branch of radial solutions $(N(m), a(m), m)$ appears in the reduced manifold as the "trivial branch" $\mathbf{r}(\mathbf{0}, m) \equiv \mathbf{0}$. A computation will show

$$
\mathbf{r}_{s}(\mathbf{0}, m)=\lambda_{l, 0}(m) I_{\mathbb{R}^{n}} .
$$

The group action $\left\{\mathfrak{R}_{T}: T \in O(d, \mathbb{R})\right\}$ on $X$ induces an action on the eigenspaces of $\mathfrak{f}_{N}(N(m), a(m), m)$ and thus on $\mathbb{R}^{n}$ via the identification $\mathbf{s} \in \mathbb{R}^{n} \longleftrightarrow \sum s_{j} \phi_{j}$. One easily verifies that our construction of the reduced manifold respects this group action, and that the map $\mathbf{s} \mapsto \mathbf{r}(\mathbf{s}, m)$ is equivariant. The theory in $[16$ therefore applies to the reduced equation. In particular we can use Vanderbauwhede and Cicogna's trick [16. Theorem 3.3, p.82] which tells us that some bifurcation does occur whenever the eigenvalue $\lambda_{l 0}(m)$ changes its sign if $m$ crosses $m_{*}$.

8.13. Application of the Implicit Function Theorem to $\mathfrak{h}$. We will show that the range of $\mathfrak{h}_{N^{\prime}}$, the range of $\mathfrak{h}_{\mathbf{r}}$, and the vector $\mathfrak{h}_{a^{\prime}}$ at $N^{\prime}=0, \mathbf{r}=\mathbf{s}=0, m=m_{*}$, and $a^{\prime}=0$ span all of $X \oplus \mathbb{R}^{n}$.

The range of $\mathfrak{h}_{N^{\prime}}$ is given by (range $\left.\mathfrak{f}_{N}\right) \oplus \mathbb{R}^{n}$. Indeed, one can split any $\delta N^{\prime}$ into a component $\delta N^{\prime \prime}$ for which $\left\langle\psi_{j}, N^{\prime \prime}\right\rangle=0$ for $j=1, \ldots, n$ and a linear combination $t_{1} \phi_{1}+\cdots+t_{n} \phi_{n}$. One then has

$$
\mathfrak{h}_{N^{\prime}} \cdot \delta N^{\prime}=\left(\begin{array}{c}
\mathfrak{f}_{N} \cdot \delta N^{\prime \prime} \\
t_{1} \\
\vdots \\
t_{n}
\end{array}\right) .
$$

The top entry can be anything in the range of $\mathfrak{f}_{N}$, and we can also arbitrarily prescribe the $t_{i}$.

The range of $\mathfrak{h}_{\mathbf{r}}$ is $\operatorname{Span}\left\{\phi_{1}, \ldots, \phi_{n}\right\} \oplus\{\mathbf{0}\}$ which is orthogonal (in the $Z_{\sigma}$ inner product) to the range of $\mathfrak{h}_{N^{\prime}}$. Adding dimensions we see that range $\mathfrak{h}_{N^{\prime}} \oplus$ range $\mathfrak{h}_{\mathbf{r}}$ has codimension one. The missing direction is provided by

$$
\mathfrak{h}_{a^{\prime}}=\left(\begin{array}{c}
\mathfrak{f}_{a} \\
0 \\
\vdots \\
0
\end{array}\right)
$$

Thus we can indeed apply the Implicit Function Theorem to $\mathfrak{h}$ at $\left(0,0,0, m_{*}, 0\right)$.

8.14. Computation of $\mathbf{r}_{s}(\mathbf{0}, m)$. Differentiation of the equations in the system (8.18) with respect to $s_{i}$ shows that at $\mathbf{s}=\mathbf{0}, \mathbf{r}=\mathbf{0}, N^{\prime}=0, a^{\prime}=0$, and for any $m$ close to $m_{*}$ one has

$$
\left\langle\psi_{k}, \frac{\partial N^{\prime}}{\partial s_{i}}\right\rangle=0
$$

One also finds

$$
\mathfrak{f}_{N} \cdot\left(\frac{\partial N^{\prime}}{\partial s_{i}}+\phi_{i}\right)+\mathfrak{f}_{a}+\sum \frac{\partial r_{k}}{\partial s_{i}} \phi_{k}=0
$$


to which we apply $\psi_{k}$,

$$
\left\langle\psi_{k}, \mathfrak{f}_{N} \cdot\left(\frac{\partial N^{\prime}}{\partial s_{i}}+\phi_{i}\right)\right\rangle+\left\langle\psi_{k}, \mathfrak{f}_{a}\right\rangle+\sum \frac{\partial r_{\bar{k}}}{\partial s_{i}}\left\langle\psi_{k}, \phi_{\bar{k}}\right\rangle=0 .
$$

Since $\psi_{k}$ vanishes on $X^{\mathrm{rad}}$ and $\mathfrak{f}_{a} \in X^{\mathrm{rad}}$, we can use (8.16) to get

$$
\left\langle\mathfrak{f}_{N}^{*} \cdot \psi_{k}, \frac{\partial N^{\prime}}{\partial s_{i}}+\phi_{i}\right\rangle+\frac{\partial r_{k}}{\partial s_{i}}=0
$$

Hence

$$
\begin{aligned}
\frac{\partial r_{k}}{\partial s_{i}} & =-\lambda_{j 0}(m)\left\langle\psi_{k}, \frac{\partial N^{\prime}}{\partial s_{i}}+\phi_{i}\right\rangle \\
& =-\lambda_{j 0}(m) \delta_{i k}-\lambda_{j 0}(m)\left\langle\psi_{k}, \frac{\partial N^{\prime}}{\partial s_{i}}\right\rangle \\
& =-\lambda_{j 0}(m) \delta_{i k}
\end{aligned}
$$

by 8.21.

8.15. A sufficient condition for bifurcation. If $\lambda_{l 0}\left(m_{*}\right)=0$, then it still need not be true that a bifurcation occurs at $m=m_{*}$, by which we mean that there exist solutions other than the radial solutions in any neighborhood of $\left(N_{*}, a_{*}, m_{*}\right)$ (this is the weakest possible interpretation of the term "bifurcation"). Vanderbauwhede and Cicogna [16, p.82] observed that one can obtain bifurcations if $\lambda_{l 0}(m)$ changes sign at $m=m_{*}$. To get such a bifurcation one must find a subgroup $G \subset O(d, \mathbb{R})$ which in its action on $\mathbb{R}^{n}$ has a one-dimensional fixed point $\operatorname{set} \operatorname{Fix}\left(G, \mathbb{R}^{n}\right)$, where $n=n_{l d}$ for short. The equivariance of the map $\mathbf{s} \rightarrow \mathbf{r}(\mathbf{s}, m)$ with respect to the action of $O(d, \mathbb{R})$ and hence of $G$ implies that $\mathbf{r} \operatorname{maps} \operatorname{Fix}\left(G, \mathbb{R}^{n}\right)$ to itself. If no bifurcation takes place at $m=m_{*}$, then the local degree at $\mathbf{s}=0$ of the map $\mathbf{s} \rightarrow \mathbf{r}(\mathbf{s}, m)$ on $\operatorname{Fix}\left(G, \mathbb{R}^{n}\right)$ must be independent of $m$. However, this degree is \pm 1 , depending on the sign of $\lambda_{l 0}(m)$. Thus if the eigenvalue changes its sign a bifurcation must occur.

This argument also works if the fixed-point space $\operatorname{Fix}\left(G, \mathbb{R}^{n}\right)$ is merely odddimensional instead of one-dimensional (the local degree of the map $\mathbf{s} \rightarrow \mathbf{r}(\mathbf{s}, m)$ on $\operatorname{Fix}\left(G, \mathbb{R}^{n}\right)$ is $(-1)^{\nu}$, where $\nu$ is the dimension of $\left.\operatorname{Fix}\left(G, \mathbb{R}^{n}\right)\right)$.

Vanderbauwhede and Cicogna [16, p.82] also proved that a "clean" bifurcation must occur, i.e. that the extra, non-symmetric solutions lie on a smooth branch, if one adds the non-degeneracy assumption

$$
\frac{d \lambda_{l 0}}{d m} \neq 0
$$

Below we will choose a specific group $G$ and verify that for large enough $l$ the eigenvalue $\lambda_{l 0}(m)$ does indeed change its sign as $m$ varies. It seems that verification of the condition (8.22) will be difficult. Nonetheless, numerical studies reported in [10] indicate that for each $l \geq 3$ exactly one bifurcation occurs, and that the bifurcating branch is a simple smooth curve. See Figure 2 .

8.16. First example of a $G$ with one-dimensional $\operatorname{Fix}\left(G, \mathbb{R}^{n_{l d}}\right)$. We can identify $\mathbb{R}^{n_{l d}}$ with the space $H_{l}\left(S^{d-1}\right)$ of spherical harmonics of degree $l$ via

$$
\left(s_{i}\right) \in \mathbb{R}^{n_{l d}} \leftrightarrow \Phi(\eta) \sum_{i=1}^{n_{l d}} s_{i} y_{l}^{(i)}(\theta) \in \operatorname{ker} \mathfrak{f}_{N} \mid Y^{l} \leftrightarrow \sum_{i=1}^{n_{l d}} s_{i} y_{l}^{(i)}(\theta) \in H_{l}\left(S^{d-1}\right)
$$


With this identification the $O(d, \mathbb{R})$ action coincides with the standard action on $H_{l}\left(S^{d-1}\right)$. If we let $G$ be the subgroup of rotations and reflections which leave the $x_{1}$-axis of $\mathbb{R}^{d}$ fixed, i.e.

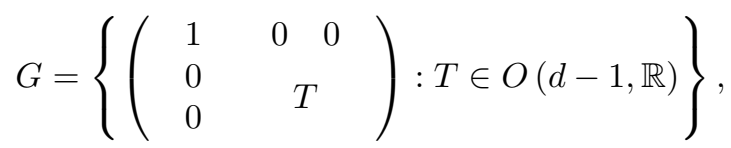

then the fixed point space of the $G$ action on $H_{l}\left(S^{d-1}\right)$ consists of those harmonics $y(\theta)$ which only depend on the $x_{1}$ variable. This space is known to be one dimensional. In the case $d=2$ it is spanned by $y(\theta)=\cos l \omega\left(\right.$ with $\left.\theta=e^{i \omega}\right)$. For $d=3$ one can choose spherical coordinates (latitude $\vartheta$ and longitude $\varphi$ ) on the sphere $S^{2}$. In these coordinates the spherical harmonic of degree $l$ invariant under $G$ is $P_{l}(\cos \vartheta)$, where $P_{l}(x)$ is the Legendre polynomial of degree $l$.

8.17. Second example of a $G$ with one-dimensional $\operatorname{Fix}\left(G, \mathbb{R}^{n_{l d}}\right)$. We begin by taking $d=2$ in our previous example, so that $n_{l d}=2$ for all $l \geq 1$. The "zonal harmonics" of degree $l$, i.e. the harmonics of degree $l$ which are also invariant under the $O(1, \mathbb{R})$ action, are all multiples of $\cos l \omega$, if $\theta=e^{i \omega}$. These functions can however also be characterized as the harmonics of degree $l$ which are invariant under the action of $D_{l}$, where $D_{l}$ is the dihedral group of order $l$. The group $D_{l}$ is generated by reflection in the $x_{1}$-axis (i.e. by $O(1, \mathbb{R})$ ) and rotation over an angle of $\frac{2 \pi}{l}$. Thus we can take $G=D_{l}$, and in this case we obtain solutions which have discrete rotational $\left(D_{l}\right)$ symmetry.

\section{The Sequence of Bifurcations as $m \rightarrow 1$}

9.1. The AG solution. To construct radial self-similar solutions Aronson and Graveleau started from equation (2.1) and considered the quantities

$$
\varphi=\frac{V}{r^{2}}, \quad \xi=r \frac{d \varphi}{d r}
$$

They observed that $\varphi$ and $\xi$ satisfy the following system of oDEs:

$$
\begin{aligned}
& (m-1) r \varphi \frac{d \varphi}{d r}=(m-1) \xi \varphi, \\
& (m-1) r \varphi \frac{d \xi}{d r}=a \xi+\varphi-(\xi+2 \varphi)^{2}-(m-1)\left((d-2) \xi \varphi+2 d \varphi^{2}\right) .
\end{aligned}
$$

In the new time variable $\tau=\int \frac{d r}{(m-1) r \varphi}$ this system becomes autonomous, so one can use phase plane techniques to analyze it.

The system has three critical points (see Figure 3): a degenerate saddle point $O$ at the origin, a hyperbolic saddle point $H$ at $(0, a)$, and another critical point $S$ at $\left((4+2 d(m-1))^{-1}, 0\right)$; the latter is a source for $a$ near 1 . As $a$ decreases toward $\frac{1}{2}$ it undergoes a Hopf bifurcation and becomes a sink. It was shown in [6] that for any $m \in(1, \infty)$ a unique value of $a=a(m) \in\left(\frac{1}{2}, 1\right)$ exists at which the unstable manifold of the point $(0, a)$ meets the center-stable manifold of the origin. This connecting orbit generates the unique radial self-similar solution, which one can find by integrating a third (trivial) equation

$$
(m-1) r \varphi \frac{d r}{d r}=(m-1) r \varphi
$$

along with (9.1) and (9.2) and plotting $V=\varphi r^{2}$ against $r$. 


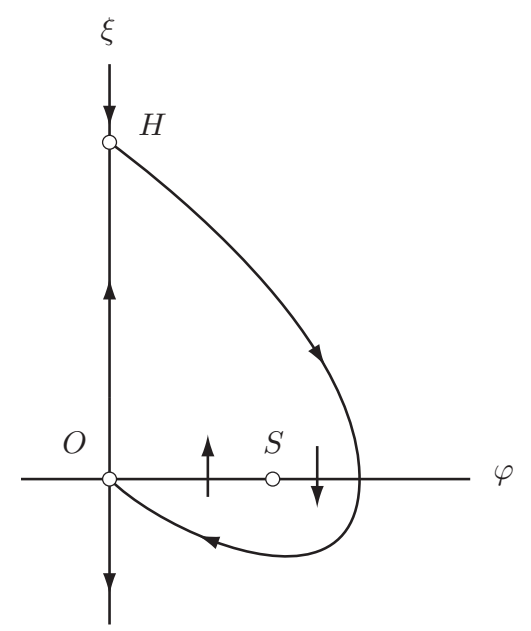

Figure 3. The AG phase plane

From the phase plane analysis in 6] (see Figure 3) it is clear that the quantity $\xi$ has exactly one zero along the connecting orbit, namely when this orbit crosses the $\varphi$ axis. We now compute, using $V=\eta$ and $r=e^{P}$,

$$
\begin{aligned}
\xi & =\frac{1}{r} \frac{d V}{d r}-2 \frac{V}{r^{2}} \\
& =\frac{1}{r} \frac{d V}{d r}\left(1-2 \frac{V}{r} \frac{d r}{d V}\right) \\
& =\frac{1}{r} \frac{d V}{d r}\left(1-2 \eta \frac{d P}{d \eta}\right) .
\end{aligned}
$$

Since $\frac{1}{r} \frac{d V}{d r}>0$ along the orbit, we find that $\psi(\eta)=1-2 \eta P^{\prime}(\eta)$ has exactly one zero, as we claimed in Lemma 8.7

9.2. The AG solution for $m \approx 1$. One can take the limit $m \rightarrow 1$ in the system (9.1), (9.2) keeping the same time variable $\tau$. This results in the system

$$
\frac{d \varphi}{d \tau}=0, \quad \frac{d \xi}{d \tau}=a \xi+\varphi-(\xi+2 \varphi)^{2} .
$$

The phase plane of (9.1), (9.2) is determined by a fast-slow system of which (9.3) is the fast part. In this phase plane all orbits converge to a point on the parabola given by $a \xi+\varphi-(\xi+2 \varphi)^{2}=0$. After that they undergo a slow evolution given by $\frac{d \varphi}{d \tau}=(m-1) \xi \varphi$. By analyzing this slow evolution one sees that the only possible value of $a \in\left[\frac{1}{2}, 1\right]$ for which stable and unstable manifolds of the origin and $(0, a)$ meet is $a=1$; for all other values of $a$ the intersection of the parabola and the $\varphi$-axis is the sink, and the unstable manifold gets trapped at this sink before it can meet the stable manifold. Aronson, Gil, and Vazquez [5] gave a rigorous proof of the following

Theorem 9.1. Let $V_{m}(r)$ be the radial self-similar solution, normalized by $\operatorname{supp} V_{m}$ $=[1, \infty)$, and let $a_{m}$ be its exponent. Then

$$
\lim _{m \searrow 1} a_{m}=1
$$


and

$$
\lim _{m \searrow 1} V_{m}(r)=r-1 .
$$

Here the convergence is in $C_{\mathrm{loc}}^{\infty}((1, \infty))$.

A corollary to this is that the corresponding function $P_{m}(\eta)$ converges in $C_{\mathrm{loc}}^{\infty}((0, \infty))$ to

$$
\bar{P}(\eta)=\lim _{m \searrow 1} P_{m}(\eta)=\ln (1+\eta) .
$$

9.3. The eigenvalues $\lambda_{l 0}(m)$ for $m \approx 1$. The following theorem illustrates the principal cause of the sequence of symmetry breaking bifurcations announced in Theorem 1.1.

Theorem 9.2. For any $l \geq 2$ an $m_{l} \in(1, \infty)$ exists such that $\lambda_{l, 0}(m)>0$ for $1<m \leq m_{l}$.

Let us choose some $\bar{m}>1$. Then only a finite number of $\lambda_{l, 0}(\bar{m})$ are non-negative (those with $l \leq l_{*}(\bar{m})$ ). This implies that for every $l>l_{*}(\bar{m})$ an $m_{l}^{*} \in(1, \bar{m})$ exists such that $\lambda_{l, 0}(m)$ changes sign at $m=m_{l}^{*}$. At each $m=m_{l}^{*}$ a symmetry breaking bifurcation must therefore occur.

Proof of Theorem 9.2. We consider the ODE (8.15) with $\lambda=0$. From the explicit forms of the coefficients $A, B$, and $C$ we see that as $m \searrow 1$

$$
\begin{aligned}
A(\eta) & \rightarrow \eta(1+\eta)^{2}, \\
(m-1) B(\eta) & \rightarrow(1-\eta)(1+\eta)^{2}, \\
(m-1) C(\eta) & \rightarrow 2(1+\eta)
\end{aligned}
$$

with convergence in $C_{\mathrm{loc}}^{\infty}((0, \infty))$. For all $m$ sufficiently close to $m=1$ the coefficient $B(\eta)$ therefore has a zero at some $\eta_{m}=1+o(1)$. We introduce a new variable

$$
\zeta=\frac{\eta-\eta_{m}}{\sqrt{m-1}}
$$

and consider the ODE (8.15) for $\Phi$ in this variable

$$
\frac{A(\eta)}{m-1} \frac{d^{2} \Phi}{d \zeta^{2}}+\frac{B(\eta)}{\sqrt{m-1}} \frac{d \Phi}{d \zeta}+\{C(\eta)-l(l+d-2) \eta\} \Phi=0 .
$$

If one multiplies this equation with $m-1$ and expands $B$ in a Taylor series at $\eta=\eta_{m}$, the coefficients of the new equation written in terms of $\zeta$ converge in $C_{\text {loc }}^{\infty}(\mathbb{R})$ to those of the equation

$$
\frac{d^{2} \Phi}{d \zeta^{2}}-\zeta \frac{d \Phi}{d \zeta}+\Phi=0
$$

The solution $\Phi(\zeta)$ with $\Phi(0)=1$ and $\Phi^{\prime}(0)=0$ is easily computed using the power series method. One finds

$$
\begin{aligned}
\Phi(\zeta) & =\sum_{n=0}^{\infty} \frac{-1}{2 n-1} \frac{\zeta^{2 n}}{2^{n} n !} \\
& =1-\frac{\zeta^{2}}{2}-\frac{1}{3} \frac{\zeta^{4}}{4 \cdot 2}-\frac{1}{5} \frac{\zeta^{6}}{6 \cdot 4 \cdot 2}-\frac{1}{7} \frac{\zeta^{8}}{8 \cdot 6 \cdot 4 \cdot 2} \cdots
\end{aligned}
$$


which happens to have two zeros at $\zeta= \pm \bar{\zeta}$ for some $0<\bar{\zeta}<\sqrt{ } 2$. It follows that the solution $\Phi_{m}(\eta)$ to (8.15) with $\Phi_{m}\left(\eta_{m}\right)=1$ and $\Phi_{m}^{\prime}\left(\eta_{m}\right)=0$ also has two zeros, approximately at $\eta=\eta_{m} \pm \sqrt{m-1}$. By the Sturm-Liouville oscillation theory any solution of (8.15) with $\lambda \leq 0$ must have a zero in the interval spanned by the zeros of $\Phi_{m}$. Since the eigenfunction corresponding to the principal eigenvalue $\lambda_{l, 0}(m)$ is positive, we must have $\lambda_{l, 0}(m)>0$, as claimed.

\section{OPEN QUESTIONS}

Our main result and the numerical studies in 10, 11 raise a number of questions which merit further study. The numerical experiments strongly support positive answers to all these questions. (See Figure 2)

Concerning the eigenvalues $\lambda_{l 0}(m)$, one would like to show:

Absence of bifurcation for $l=2$. In other words, one would like to prove $\lambda_{20}(m)>0$ for all $m$.

Bifurcation for all $l \geq 3$. That is, for each $l>2$ one has $\lambda_{l 0}(m)<0$ for all sufficiently large $m$.

Monotonicity of the eigenvalue and simplicity of the bifurcations. That is,

$$
\frac{d \lambda_{l 0}(m)}{d m}<0
$$

at least for all $m$ with $\lambda_{l 0}(m)=0$. This would imply that there is at most one bifurcation for each $l$, and together with the previous two items, that a unique and simple bifurcation occurs for each $l \geq 3$.

The above problems require analysis of the eigenvalue equations defining the $\lambda_{l j}(m)$, and thus are ODE problems. In contrast, the following are PDE problems:

Global continuation. The self-similar solutions $(V, a, m)$ we construct in this paper exist in a neighborhood of each of the bifurcation points $\left(V_{m_{l}}, a_{m_{l}}, m_{l}\right)$. Can one prove a global bifurcation theorem, and, in particular, can one show that the bifurcating branches extend to a family of solutions $\left\{\left(V_{m}, a_{m}, m\right) \mid 1<m<m_{l}\right\}$ ?

Given the self-similar solutions there are a number of natural questions about the dynamics of hole filling:

Dynamical instability of non-radially symmetric self-similar solutions: Show that for a generic initial condition (in the sense of Baire category) $v(x, y, 0)$ the asymptotics of "hole filling" is not given by any self-similar solution.

Dynamical stability within the symmetry class in $d=2$. The self-similar solutions in $d=2$ have $l$-fold dihedral $\left(D_{l}\right)$ symmetry. Show that for any solution whose initial data have $\left(D_{l}\right)$-symmetry the asymptotics of "hole filling" is given by a self-similar solution with $\left(D_{l}\right)$-symmetry, in the same way that the AG solution describes hole filling for axially symmetric solutions [1].

\section{ACKNOWLEDGEMENTS}

We are indebted to the members of the Gratton group in Tandil and Buenos Aires for the gravity current experiments, numerical results, and helpful discussions. We are particularly indebted to Santiago Betelú for his numerical work on the bifurcation and intermediate asymptotics problems, and for permission to include the bifurcation diagram figure from [10] in this paper. 


\section{REFERENCES}

[1] S.B.Angenent and D.G.Aronson, The focusing problem for the radially symmetric porous medium equation, Comm. P.D.E. 20 (1995), 1217-1240. MR 96c:35074

[2] S.B.Angenent and D.G.Aronson, Intermediate asymptotics for convergent viscous gravity currents, Phys. Fluids 7 (1995), 223-225. MR 95h:76038

[3] D.G.Aronson and Ph.Benilan, Regularité des solutions de l'équation des milieux poreux dans $\mathbb{R}^{N}$, C.R. Acad. Sci. Paris 288 (1979), 103-105. MR 82i:35090

[4] D.G.Aronson, The porous medium equation, in Some Problems in Nonlinear Diffusion, edited by A.Fasano and M.Primicerio, Lecture Notes in Mathematics 1224, Springer, Berlin, 1986. MR 88a:35130

[5] D.G.Aronson, O.Gil, and J.L.Vazquez, Limit behaviour of focusing solutions to nonlinear diffusions, Comm. P.D.E. 23 (1998), 307-332. MR 98j:35082

[6] D.G.Aronson and J. Graveleau, A self-similar solution to the focusing problem for the porous medium equation, Euro. J. Appl. Math. 4 (1993), 65-81. MR 94c:35096

[7] G.I.Barenblatt, Scaling, Self-similarity and Intermediate Asymptotics, Cambridge University Press, New York, 1998. MR 98a:00005

[8] C.M.Bender and S.A.Orszag, Advanced Mathematical Methods for Scientists and EnGineERs, International Series in Pure and Applied Mathematics, McGraw-Hill Book Co., New York, 1978. MR 80d:00030

[9] J.Bergh and J.Löffström, Interpolation Spaces, Grundlehren der mathematischen Wissenschaften 223, Springer Verlag, 1976. MR 58:2349

[10] S.I.Betelú, D.G.Aronson, and S.B.Angenent, Renormalization study of two-dimensional convergent solutions of the porous medium equation, Physica D, 138 (2000), no. 3-4, 344-359. (Preprint: patt-sol 9908006 from http://xxx.lanl.gov/.) MR 2000j:76132

[11] S.I.Betelú, J.Lowengrub, and D.G.Aronson, Focusing of an elongated hole in porous medium flow, preprint.

[12] S.N.Chow and J.K.Hale, Methods of Bifurcation Theory, Grundlehren der mathematischen Wissenschaften 251, Springer, 1982. MR 84e:58019

[13] J.A.Diez, L.P.Thomas, S.Betelú, R.Gratton, B.Marino, J.Gratton, D.G.Aronson, and S.B.Angenent, Noncircular converging flows in viscous gravity currents, Phys. Rev. E 58(1998), 6182-6187.

[14] G.B.Folland, Introduction to Partial Differential Equations, Princeton University Press, 1976. MR 58:29031

[15] D.Gilbarg and N.S.Trudinger, Elliptic Partial Differential Equations of Second OrDER, 2nd edition, Grundlehren der mathematischen Wissenschaften 224, Springer Verlag, 1983. MR 86c:35035

[16] M.Golubitsky, I.Stewart, and D.G.Schaeffer, Singularities and Groups in Bifurcation Theory, volume II, Springer Verlag, Applied Math.Sciences 69 (1988). MR 89m:58038

[17] J.Graveleau, Quelques solutions auto-semblables pour l'équation dela chaleur non- linéair, Rapport Interne C.E.A. (1972).

[18] H.Hochstadt, The Functions of Mathematical Physics, Dover, 1986. MR 88b:33001

[19] L.Hörmander, The Analysis of Linear Partial Differential Operators (I), Springer Grundlehren Bd.256., 1983. MR 85g:35002a

[20] T.Kato, Perturbation Theory for Linear Operators. Second edition. Grundlehren der Mathematischen Wissenschaften 132, Springer-Verlag, Berlin-New York, 1976. MR 53:11389

[21] G.da Prato and P.Grisvard, Sommes d'opérateurs linéaires et équations différentielles opérationnelles, J. Math. Pures Appl. (9) 54 (1975), no. 3, 305-387. MR 56:1129

[22] E.M.Stein, Harmonic Analysis, Princeton Mathematical Series 43, P.U.P. 1993. MR 95c: 42002

[23] E.T.Whittaker and G.N.Watson, A Course in Modern Analysis, Cambridge University Press, 1927. MR 97k:01072 (Review of the reprint of the fourth (1927) edition.) 53706

Department of Mathematics, University of Wisconsin-Madison, Madison, Wisconsin

E-mail address: angenent@math.wisc.edu

School of Mathematics, University of Minnesota, Minneapolis, Minnesota 55455

E-mail address: don@math.umn.edu 